\title{
Representations of spin quiver Hecke algebras for orthosymplectic Lie superalgebras
}

\author{
Konstantina Christodoulopoulou ${ }^{\mathrm{a}}$, Kyu-Hwan Lee ${ }^{\mathrm{b}, *, 1}$ \\ a Department of Mathematics, University of Florida, Gainesville, FL 32611-8105, USA \\ b Department of Mathematics, University of Connecticut, Storrs, CT 06269-3009, USA
}

\section{A R T I C L E I N F O}

\section{Article history:}

Received 21 September 2015

Received in revised form 1 April

2016

Available online 11 May 2016

Communicated by D. Nakano

\begin{abstract}
A B S T R A C T
\end{abstract}
We construct all the irreducible representations of spin quiver Hecke algebras for orthosymplectic Lie superalgebras $\operatorname{osp}(1 \mid 2 n)$, and show that their highest weights are given by the dominant words. We use the dominant Lyndon words to construct the cuspidal modules and show that the irreducible representations are the simple heads of standard representations constructed by induction from the cuspidal modules.

() 2016 Elsevier B.V. All rights reserved.

\section{Introduction}

Introduced by Khovanov and Lauda [11] and independently by Rouquier [16], the Khovanov-LaudaRouquier (KLR) algebras (also known as quiver Hecke algebras) have attracted much attention as these algebras categorify the lower (or upper) half of a quantum group. More precisely, the Cartan datum associated with a Kac-Moody algebra $\mathfrak{g}$ gives rise to a KLR algebra $R$. The category of finitely generated projective graded modules of this algebra can be given a bialgebra structure by taking the Grothendieck group, and taking the induction and restriction functors as multiplication and co-multiplication. It turns out that this bialgebra is isomorphic to Lusztig's integral form of $U_{q}^{-}(\mathfrak{g})$, and in this sense we say that the KLR algebra $R$ categorifies the negative part $U_{q}^{-}(\mathfrak{g})$ of the quantum group.

In the study of the category of representations, it is of fundamental interest to construct irreducible representations of $R$. In the paper [12], Kleshchev and Ram defined a class of cuspidal representations for finite types, showed that every irreducible representation appears as the head of some induction of these cuspidal modules, and constructed almost all cuspidal representations. Hill, Melvin, and Mondragon in [6]

\footnotetext{
* Corresponding author.

E-mail addresses: kchristod@ufl.edu (K. Christodoulopoulou),khlee@math.uconn.edu (K.-H. Lee).

1 This work was partially supported by a grant from the Simons Foundation (\#318706).
} 
completed the construction of cuspidal representations in all finite types, and re-framed them in a more unified manner. Using a different approach, Benkart, Kang, Oh, and Park in [2] also constructed irreducible representations utilizing a crystal structure on the isomorphism classes of irreducible representations of a KLR algebra obtained by Lauda and Vazirani in [13].

Along these developments, the case of Kac-Moody superalgebras has been considered. As a foundational work in the superalgebra case, Kang, Kashiwara, and Tsuchioka generalized the KLR algebras to the spin quiver Hecke algebras [10]. Subsequently, Hill and Wang [7] and Kang, Kashiwara, and Oh [8,9] showed that the spin quiver Hecke algebras provide a categorification of half of quantum Kac-Moody superalgebras without isotropic roots. It is well known that a Kac-Moody superalgebra can be associated to a generalized Cartan matrix. The only finite-dimensional Kac-Moody superalgebras, which are not Lie algebras, are the orthosymplectic Lie superalgebras $\operatorname{osp}(1 \mid 2 n)$. Naturally, it is an important task to construct all the irreducible representations of the spin quiver Hecke algebras corresponding to $\operatorname{osp}(1 \mid 2 n)$.

In this paper, we construct all the irreducible representations of spin quiver Hecke algebras for orthosymplectic Lie superalgebras $\operatorname{osp}(1 \mid 2 n)$. Our method is similar to that of Kleshchev and Ram [12] and is based on the work of Clark, Hill, and Wang [5] on quantum shuffles and dominant Lyndon words. Both of these papers are closely related in the work of Leclerc [14]. We present an explicit construction of cuspidal representations in Proposition 3.2 and use the cuspidal representations as building blocks to obtain other irreducible representations. In this process, the computation of the leading coefficients of canonical basis elements is crucial and requires a careful analysis of signs and degrees for the corresponding representations of the spin quiver Hecke algebra in categorification. With cuspidal representations at hand, we construct standard representations through induction from cuspidal representations, and show that they have irreducible heads. Finally, as the main result (Theorem 4.5) of this paper, we prove that these irreducible heads form a complete set of irreducible representations of the spin quiver Hecke algebra for $o s p(1 \mid 2 n)$.

With the results of this paper, we can consider some future directions. First, as in [4], one can use a general convex order to construct standard representations and study their homological properties. Next, one can obtain a concrete crystal structure on the category of representations of $o s p(1 \mid 2 n)$, following [13] and $[9]$. We hope that these directions may be pursed in the near future.

The outline of this paper is as follows. In Section 1, we fix notations for $\operatorname{osp}(1 \mid 2 n)$, consider quantum shuffle products and combinatorics of Lyndon words, and recall the construction of the canonical basis. In Section 2, spin quiver Hecke algebras are introduced and properties of their representations are presented. The next section is devoted to the construction of cuspidal representations of the spin quiver Hecke algebras. In the last section, we construct standard representations and obtain all the irreducible representations.

\section{Quantum superalgebras and canonical bases}

\subsection{Root data}

Let $I=I_{\overline{0}} \cup I_{\overline{1}}$ be a $\mathbb{Z} / 2 \mathbb{Z}$-graded finite set of size $n$, and let $p: I \rightarrow\{\overline{0}, \overline{1}\}$ be the corresponding parity function. We assume that $I_{\overline{1}} \neq \emptyset$. Consider a generalized Cartan matrix $A=\left(a_{i j}\right)_{i, j \in I}$ such that $(\mathrm{C} 1) a_{i i}=2$ for each $i \in I ;(\mathrm{C} 2) a_{i j} \in \mathbb{Z}_{\leq 0}$ for $i \neq j$; (C3) $a_{i j}=0$ if and only if $a_{j i}=0$; (C4) $a_{i j} \in 2 \mathbb{Z}$ for $i \in I_{\overline{1}}$ and $j \in I$. We assume that the matrix $A$ is symmetrizable, i.e. there exists an invertible matrix $D=\operatorname{diag}\left(s_{1}, \ldots, s_{n}\right)$ with $D A$ symmetric. Furthermore, we choose $D$ such that $s_{i} \in \mathbb{Z}_{>0}$ and $\operatorname{gcd}\left(s_{1}, \ldots, s_{n}\right)=1$, and assume that the integer $s_{i}$ is odd if and only if $i \in I_{\overline{1}}$.

In this paper, we will be primarily interested in the following case: the index set $I=\{1,2, \ldots, n\}$ with $I_{\overline{1}}=\{n\}$, 


$$
A=\left(\begin{array}{ccccccc}
2 & -1 & 0 & \cdots & 0 & 0 & 0 \\
-1 & 2 & -1 & \cdots & 0 & 0 & 0 \\
0 & -1 & 2 & \cdots & 0 & 0 & 0 \\
\vdots & \vdots & \vdots & \vdots & \vdots & \vdots & \vdots \\
0 & 0 & 0 & \cdots & -1 & 2 & -1 \\
0 & 0 & 0 & \cdots & 0 & -2 & 2
\end{array}\right)
$$

and $D=(2,2, \ldots, 2,1)$. Throughout this paper, we let $\mathfrak{g}$ be the Kac-Moody superalgebra associated to a symmetrizable generalized Cartan matrix $A$ as in (1.1) and let $U_{q}(\mathfrak{g})$ be the corresponding quantized enveloping superalgebra defined as in [1]. The generators of $\mathfrak{g}$ will be denoted by $e_{i}, f_{i}$ and $h_{i}(i \in I)$. The subalgebra of $U_{q}(\mathfrak{g})$ generated by the elements $e_{i}(i \in I)$ will be denoted by $U_{q}^{+}$. Let $\widetilde{\Phi}=\widetilde{\Phi}_{\overline{0}} \cup \widetilde{\Phi}_{\overline{1}}$ be the root system for $\mathfrak{g}$ and let

$$
\Phi=\Phi_{\overline{0}} \cup \Phi_{\overline{1}}=\left\{\beta \in \widetilde{\Phi} \mid \frac{1}{2} \beta \notin \widetilde{\Phi}\right\}
$$

be the reduced root system for $\mathfrak{g}$, where $\Phi_{s}=\Phi \cap \widetilde{\Phi}_{s}$ for $s \in\{\overline{0}, \overline{1}\}$. Denote the set of simple roots by $\Pi=\Pi_{\overline{0}} \cup \Pi_{\overline{1}}=\left\{\alpha_{i} \mid i \in I\right\}$ and the set of positive roots by $\widetilde{\Phi}^{+}$. Then we put $\Phi^{+}=\Phi \cap \widetilde{\Phi}^{+}$. We also have the corresponding sets $\widetilde{\Phi}_{\overline{0}}^{+}, \Phi_{\overline{0}}^{+}$(resp. $\widetilde{\Phi}_{\overline{1}}^{+}, \Phi_{\overline{1}}^{+}$) of positive even (resp. odd) roots. For example, when $n=2$, we have $I_{\overline{1}}=\{2\}$ and

$$
\begin{array}{ll}
\widetilde{\Phi}^{+}=\left\{\alpha_{1}, \alpha_{2}, \alpha_{1}+\alpha_{2}, \alpha_{1}+2 \alpha_{2}, 2 \alpha_{2}, 2 \alpha_{1}+2 \alpha_{2}\right\}, & \Phi^{+}=\left\{\alpha_{1}, \alpha_{2}, \alpha_{1}+\alpha_{2}, \alpha_{1}+2 \alpha_{2}\right\}, \\
\widetilde{\Phi}_{\overline{0}}^{+}=\left\{\alpha_{1}, \alpha_{1}+2 \alpha_{2}, 2 \alpha_{2}, 2 \alpha_{1}+2 \alpha_{2}\right\}, & \Phi_{\overline{0}}^{+}=\left\{\alpha_{1}, \alpha_{1}+2 \alpha_{2}\right\}, \\
\widetilde{\Phi}_{\overline{1}}^{+}=\Phi_{\overline{1}}^{+}=\left\{\alpha_{2}, \alpha_{1}+\alpha_{2}\right\} . &
\end{array}
$$

The $\mathbb{Z}$-lattice spanned by $\Pi$ is denoted by $Q$. We define $p\left(\alpha_{i}\right)=p(i), i \in I$, and extend it to the additive monoid $Q^{+}:=\sum_{i} \mathbb{Z}_{\geq 0} \alpha_{i}$. Define a symmetric bilinear form $(\cdot, \cdot): Q \times Q \longrightarrow \mathbb{Z}$ by $\left(\alpha_{i}, \alpha_{j}\right)=b_{i j}$, where $B=D A=\left(b_{i j}\right)$.

\subsection{Quantum shuffle superalgebras}

Let $\mathcal{W}$ be the set of words on the alphabet $I$ with the empty word $\emptyset$. An element $\mathbf{i} \in \mathcal{W}$ will be denoted by

$$
\mathbf{i}=\left(i_{1}, i_{2}, \ldots, i_{d}\right)=i_{1} i_{2} \ldots i_{d}
$$

Define $|\mathbf{i}|=\left|\left(i_{1}, \ldots, i_{d}\right)\right|=\alpha_{i_{1}}+\cdots+\alpha_{i_{d}} \in Q^{+}$and $p(\mathbf{i})=p(|\mathbf{i}|)$ for $\mathbf{i} \in \mathcal{W}$. The length of $\mathbf{i}$ will be denoted by $\ell(\mathbf{i})$, i.e. $\ell\left(i_{1}, i_{2}, \ldots, i_{d}\right)=d$. For $\alpha \in Q^{+}$, set $\mathcal{W}_{\alpha}=\{\mathbf{i} \in \mathcal{W}|| \mathbf{i} \mid=\alpha\}$. Let $\mathcal{F}$ be the free associative superalgebra over $\mathbb{Q}(q)$ generated by $I$, where $q$ is an indeterminate. Note that $\mathcal{F}$ has a weight decomposition $\mathcal{F}=\bigoplus_{\alpha \in Q^{+}} \mathcal{F}_{\alpha}$, where $\mathcal{F}_{\alpha}=\mathcal{F} \cap \mathcal{W}_{\alpha}$. The set $\mathcal{W}$ is naturally considered as a $\mathbb{Q}(q)$-linear basis of $\mathcal{F}$.

We define the quantum shuffle product $\diamond: \mathcal{F} \times \mathcal{F} \longrightarrow \mathcal{F}$ inductively by

$$
(x i) \diamond(y j)=(x \diamond(y j)) i+(-1)^{p(x i) p(j)} q^{-(|x i|,|j|)}((x i) \diamond y) j
$$

for $x, y \in \mathcal{W}$ and $i, j \in I$ and by extending it linearly, where we set $\emptyset \diamond x=x \diamond \emptyset=x$ for $x \in \mathcal{W}$.

Thus we have two different products on $\mathcal{F}$; one is juxtaposition for the free algebra structure and the other is the quantum shuffle product $\diamond$. When we consider a $k$ th power of an element $x \in \mathcal{F}$, we will write $x^{k}$ for juxtaposition and $x^{\diamond k}$ for the quantum shuffle product.

Proposition 1.3. ([5, Corollary 3.4]) There exists an algebra embedding $\Psi: U_{q}^{+} \longrightarrow(\mathcal{F}, \diamond)$ such that $\Psi\left(e_{i}\right)=i$. 
Define $\mathcal{U}=\Psi\left(U_{q}^{+}\right)$to be the subalgebra of $(\mathcal{F}, \diamond)$ generated by $I$. The algebra $\mathcal{U}$ is $Q^{+}$-graded with $\mathcal{U}_{\alpha}=\mathcal{U} \cap \mathcal{F}_{\alpha}$. We define the shuffle product on $\mathcal{F} \otimes \mathcal{F}$ by

$$
(w \otimes x) \diamond(y \otimes z)=(-1)^{p(x) p(y)} q^{-(|x|,|y|)}(w \diamond y) \otimes(x \diamond z) \quad \text { for } x, y, z, w \in \mathcal{W},
$$

and define the map $\Delta: \mathcal{F} \longrightarrow \mathcal{F} \otimes \mathcal{F}$ by

$$
\Delta\left(i_{1}, \ldots, i_{d}\right)=\sum_{0 \leq k \leq d}\left(i_{k+1}, \ldots, i_{d}\right) \otimes\left(i_{1}, \ldots, i_{k}\right)
$$

Proposition 1.4. ([5, Proposition 3.13]) There exists a nondegenerate symmetric bilinear form

$$
(\cdot, \cdot): \mathcal{U} \times \mathcal{U} \longrightarrow \mathbb{Q}(q)
$$

that satisfies the following properties:

(1) $(1,1)=1$;

(2) $(i, j)=\delta_{i j}$ for $i, j \in I$;

(3) $(x, y \diamond z)=(\Delta(x), y \otimes z)$ for $x, y, z \in \mathcal{U}$, where the induced bilinear form is given by

$$
\left(x \otimes x^{\prime}, y \otimes y^{\prime}\right):=(x, y)\left(x^{\prime}, y^{\prime}\right) .
$$

In the following proposition, we recall some linear maps on $\mathcal{F}$, which give rise to important (anti-)automorphisms on $\mathcal{U}$. For $\nu=\sum_{i} c_{i} \alpha_{i} \in Q^{+}$, let

$$
N(\nu)=\frac{1}{2}\left((\nu, \nu)-\sum_{i \in I} c_{i}\left(\alpha_{i}, \alpha_{i}\right)\right) \quad \text { and } \quad P(\nu)=\frac{1}{2}\left(p(\nu)^{2}-\sum_{i \in I} c_{i} p\left(\alpha_{i}\right)\right),
$$

where $p\left(\alpha_{i}\right) \in\{0,1\}$ and $\sum_{i \in I} c_{i} p\left(\alpha_{i}\right)$ are interpreted as integers. For any $\mathbf{i} \in \mathcal{W}$, we set $P(\mathbf{i})=P(|\mathbf{i}|)$.

Proposition 1.6. ([5, Proposition 3.10])

(1) Let $\tau: \mathcal{F} \rightarrow \mathcal{F}$ be the $\mathbb{Q}(q)$-linear map defined by

$$
\tau\left(i_{1}, \ldots, i_{d}\right)=\left(i_{d}, \ldots, i_{1}\right)
$$

Then $\tau(x \diamond y)=\tau(y) \diamond \tau(x)$ for all $x, y \in \mathcal{F}$.

(2) Let $^{-}: \mathcal{F} \rightarrow \mathcal{F}$ be the $\mathbb{Q}$-linear map defined by $\bar{q}=-q^{-1}$ and

$$
\overline{\left(i_{1}, \ldots, i_{d}\right)}=(-1)^{\sum_{s<t} p\left(i_{s}\right) p\left(i_{t}\right)} q^{-\sum_{s<t}\left(\alpha_{i_{s}}, \alpha_{i_{t}}\right)}\left(i_{d}, \ldots, i_{1}\right) .
$$

Then $\overline{x \diamond y}=\bar{x} \diamond \bar{y}$ for all $x, y \in \mathcal{F}$.

(3) Let $\sigma: \mathcal{F} \rightarrow \mathcal{F}$ be the $\mathbb{Q}$-linear map defined by $\sigma(q)=-q^{-1}$ and

$$
\sigma\left(i_{1}, \ldots, i_{d}\right)=(-1)^{\sum_{s<t} p\left(i_{s}\right) p\left(i_{t}\right)} q^{-\sum_{s<t}\left(\alpha_{i_{s}}, \alpha_{i_{t}}\right)}\left(i_{1}, \ldots, i_{d}\right) .
$$

Then $\sigma(x)=\overline{\tau(x)}, \sigma(x \diamond y)=\sigma(y) \diamond \sigma(x)$ for all $x, y \in \mathcal{F}$, and

$$
\sigma(\mathbf{i})=(-1)^{P(\mathbf{i})} q^{-N(|\mathbf{i}|)} \mathbf{i} \text { for all } \mathbf{i} \in \mathcal{W} .
$$


Remark 1.7. Since $\sum_{s<t}\left(\alpha_{i_{s}}, \alpha_{i_{t}}\right) \in 2 \mathbb{Z}$, it is easy to check from the definition that $\sigma^{2}=\operatorname{Id}_{\mathcal{F}}$.

The following lemma will be useful later.

Lemma 1.8. Let $\mu, \nu \in Q^{+}$. We have the following properties

(1) $N(\nu) \in 2 \mathbb{Z}$ for all $\nu \in Q^{+}$;

(2) $N(\mu+\nu)=N(\mu)+N(\nu)+(\mu, \nu)$;

(3) $P(\mu+\nu)=P(\mu)+P(\nu)+p(\mu) p(\nu)$.

Proof. It is easy to see from (1.5) that $N\left(\alpha_{i_{1}}+\ldots+\alpha_{i_{k}}\right)=\sum_{1 \leq s<t \leq k}\left(\alpha_{i_{s}}, \alpha_{i_{t}}\right)$. Now statement (1) follows from the fact that $\left(\alpha_{i}, \alpha_{j}\right) \in 2 \mathbb{Z}$ for all $i, j \in I$. The equalities (2) and (3) follow from (1.5) by straightforward computations.

\subsection{Dominant words and Lyndon words}

Fix a total ordering $\prec$ on $I$ to be $1 \prec 2 \prec \cdots \prec n$, and put the induced lexicographic ordering $\prec$ on $\mathcal{W}$. A word $\mathbf{i} \in \mathcal{W}$ is called dominant if $\mathbf{i}=\max (u)$ for some $u \in \mathcal{U}$. Denote the set of dominant words by $\mathcal{W}^{+}$, and define $\mathcal{W}_{\alpha}^{+}=\mathcal{W}^{+} \cap \mathcal{W}_{\alpha}$. A word $\mathbf{i}=\left(i_{1}, \ldots, i_{d}\right) \in \mathcal{W}$ is called Lyndon if it is smaller than any of its proper right factors. Let $\mathcal{L}$ be the set of Lyndon words in $\mathcal{W}$, and let $\mathcal{L}^{+}$be the set of dominant Lyndon words in $\mathcal{W}$. Recall that every word $\mathbf{i} \in \mathcal{W}$ has a canonical factorization as a product of non-increasing Lyndon words:

$$
\mathbf{i}=\mathbf{i}_{1} \cdots \mathbf{i}_{d}, \quad \mathbf{i}_{1}, \ldots, \mathbf{i}_{d} \in \mathcal{L}, \quad \mathbf{i}_{1} \succeq \cdots \succeq \mathbf{i}_{d} .
$$

Theorem 1.9. ([5, Theorem 4.8])

(1) The map $\mathbf{i} \mapsto|\mathbf{i}|$ is a bijection from $\mathcal{L}^{+}$to $\Phi^{+}$. Given $\beta \in \Phi^{+}$, we write $\iota^{+}(\beta)$ for the pre-image of $\beta$ under this bijection.

(2) Assume that $\mathbf{i}=\mathbf{i}_{1} \cdots \mathbf{i}_{d}$ is the canonical factorization. Then $\mathbf{i} \in \mathcal{W}^{+}$if and only if $\mathbf{i}_{s} \in \mathcal{L}^{+}$for each $s=1,2, \ldots, d$.

The set of dominant Lyndon words was computed in the work of Clark, Hill and Wang:

Proposition 1.10. ([5, Proposition 6.5]) The set of dominant Lyndon words for $\mathfrak{g}$ is given by

$$
\mathcal{L}^{+}=\{(i, \ldots, j) \mid 1 \leq i \leq j \leq n\} \cup\{(i, \ldots, n, n, \ldots, j) \mid 1 \leq i<j \leq n\} .
$$

Remark 1.11. As a related result, a basis for $\mathfrak{g}$ arising from Lyndon words was obtained by Bokut, Kang, Lee and Malcomson in [3].

The following corollary is similar to [12, Lemma 5.9] and slightly generalizes [5, Corollary 4.17] in our context.

Corollary 1.12. Let $\beta \in \Phi^{+}$and $m \in \mathbb{Z}_{\geq 0}$. Then $\boldsymbol{\iota}^{+}(\beta)^{m}$ is the smallest dominant word in $\mathcal{W}_{m \beta}$.

Proof. Let $\mathbf{i}=\boldsymbol{\iota}^{+}(\beta)$, and let $\mathbf{j}$ be a dominant word of weight $m \beta$ such that $\mathbf{j} \prec \mathbf{i}^{m}$. We show that this is impossible by checking the different cases for $\mathbf{i} \in \mathcal{L}^{+}$. Let $\mathbf{j}=\mathbf{j}_{1} \mathbf{j}_{2} \ldots \mathbf{j}_{s}$ be the canonical factorization of $\mathbf{j}$, 
where $\mathbf{j}_{1}, \mathbf{j}_{2}, \ldots, \mathbf{j}_{s} \in \mathcal{L}^{+}$and $\mathbf{j}_{1} \succeq \mathbf{j}_{2} \succeq \ldots \succeq \mathbf{j}_{s}$. Since $\mathbf{j} \prec \mathbf{i}^{m}$, there exists $k$ such that $\mathbf{j}_{r}=\mathbf{i}$ for $r<k$ and $\mathbf{j}_{k} \prec \mathbf{i}$ (see the discussion before Lemma 4.1 in [15]). Clearly $k \leq m$. Suppose $\mathbf{j}=\mathbf{i}^{k-1} \mathbf{j}_{k} \ldots \mathbf{j}_{s}$, where $\mathbf{i} \succ \mathbf{j}_{k} \succeq \ldots \succeq \mathbf{j}_{s}$. Set $\gamma_{s}=\left|\mathbf{j}_{s}\right|$ for all $s$. By assumption $\gamma_{1}+\ldots+\gamma_{s}=m \beta$. Recall that $\mathcal{L}^{+}=\{(i, \ldots, j) \mid 1 \leq$ $i \leq j \leq n\} \cup\{(i, \ldots, n, n, \ldots, j) \mid 1 \leq i<j \leq n\}$. Assume that $\mathbf{i}=(i, \ldots, j)$ for some $1 \leq i \leq j \leq n$. Thus $m \beta=m \alpha_{i}+\ldots+m \alpha_{j}$. Since $\mathbf{j}_{r} \prec \mathbf{i}$ for $k \leq r \leq s$, and the coefficient of $\alpha_{\ell}$ for $\ell<i$ in $m \beta$ is zero, it follows that $\mathbf{j}_{r} \in\{(i, \ldots, t) \mid i \leq t \leq j-1\}$ for all $k \leq r \leq s$, and from the coefficient of $\alpha_{i}$ in $m \beta$ we conclude that $s=m$. But then the coefficient of $\alpha_{j}$ in $\gamma_{1}+\ldots+\gamma_{s}$ will be $k-1<m$, which is a contradiction. Next, suppose that $\mathbf{i}=(i, \ldots, n, n)$ for some $1 \leq i \leq n-1$. Then $m \beta=m \alpha_{i}+\ldots+2 m \alpha_{n}$. By a similar argument it follows that $\mathbf{j}_{r} \in\{(i, \ldots, t) \mid i \leq t \leq n\}$ for $k \leq r \leq s, s=m$, and the coefficient of $\alpha_{n}$ in $\gamma_{1}+\ldots+\gamma_{s}$ is $2(k-1)<2 m$, which is again contradiction. Finally, assume that $\mathbf{i}=(i, \ldots, n, n, \ldots, j)$ for some $1 \leq i<j \leq n-1$. Then $m \beta=m \alpha_{i}+\ldots+m \alpha_{j-1}+2 m \alpha_{j}+\ldots+2 m \alpha_{n}$. Similarly as above it follows that $\mathbf{j}_{r} \in\{(i, \ldots, t) \mid i \leq t \leq n\} \cup\{(i, \ldots, n, n, \ldots, t) \mid i<t \leq j+1\}$, for $k \leq r \leq s, s=m$ and the coefficient of $\alpha_{j}$ in $\gamma_{1}+\ldots+\gamma_{s}$ will be at most $2(k-1)+m-k+1=m+k-1<2 m$, which is another contradiction.

\subsection{Maximal elements in shuffle products}

Let $\mathcal{A}=\mathbb{Z}\left[q, q^{-1}\right]$. For $\mathbf{i} \in \mathcal{L}^{+}$set $q_{\mathbf{i}}:=q^{\frac{(|\mathbf{i}|,|\mathbf{i}|)}{2}}$, and define

$$
[m]_{\mathbf{i}}=\left\{\begin{array}{ll}
\frac{q_{\mathbf{i}}^{m}-q_{\mathbf{i}}^{-m}}{q_{\mathbf{i}}-q_{\mathbf{i}}^{-1}} & \text { if } p(\mathbf{i})=\overline{0} \\
\frac{\left(-q_{\mathbf{i}}\right)^{m}-q_{\mathbf{i}}^{-m}}{-q_{\mathbf{i}}-q_{\mathbf{i}}^{-1}} & \text { if } p(\mathbf{i})=\overline{1}
\end{array} \quad \text { and } \quad[m]_{\mathbf{i}} !=[m]_{\mathbf{i}}[m-1]_{\mathbf{i}} \ldots[1]_{\mathbf{i}}\right.
$$

In particular, $[2]_{n}=-\left(q-q^{-1}\right)$,

$$
q_{\mathbf{i}}=\left\{\begin{array}{ll}
q^{2} & \text { if } p(\mathbf{i})=\overline{0}, \\
q & \text { if } p(\mathbf{i})=\overline{1},
\end{array} \quad \text { and } \quad[m]_{\mathbf{i}}= \begin{cases}\frac{q^{2 m}-q^{-2 m}}{q^{2}-q^{-2}} & \text { if } p(\mathbf{i})=\overline{0}, \\
\frac{(-q)^{m}-q^{-m}}{-q-q^{-1}} & \text { if } p(\mathbf{i})=\overline{1} .\end{cases}\right.
$$

The following lemma follows as in [12, Lemma 5.1].

Lemma 1.14. Let $w, w^{\prime}, \ell, g \in \mathcal{W}$ with $|w|=|\ell|,\left|w^{\prime}\right|=|g|, w \preceq \ell$ and $w^{\prime} \preceq g$. Then $\max \left(w \diamond w^{\prime}\right) \preceq \max (l \diamond g)$. Moreover, if $w \prec \ell$ or $w^{\prime} \prec g$, then $\max \left(w \diamond w^{\prime}\right) \prec \max (l \diamond g)$.

The next result generalizes [5, Lemma 4.5] and will be useful in computing leading coefficients in quantum shuffle products for canonical factorizations.

Lemma 1.15. Assume that $\mathbf{i} \in \mathcal{L}, \mathbf{j} \in \mathcal{W}^{+}, \mathbf{i} \succeq \mathbf{j}$ and $m \in \mathbb{Z}_{>0}$. Then $\max \left(\mathbf{i}^{m} \diamond \mathbf{j}\right)=\max \left(\mathbf{j} \diamond \mathbf{i}^{m}\right)=\mathbf{i}^{m} \mathbf{j}$ Moreover:

(1) If $\mathbf{i} \succ \mathbf{j}$, then the coefficient of $\mathbf{i}^{m} \mathbf{j}$ in $\mathbf{i}^{m} \diamond \mathbf{j}$ is $(-1)^{p(m \mathbf{i}) p(\mathbf{j})} q^{-(m|\mathbf{i}|,|\mathbf{j}|)}$

(2) If $\mathbf{i} \succ \mathbf{j}$, then the coefficient of $\mathbf{i}^{m} \mathbf{j}$ in $\mathbf{j} \diamond \mathbf{i}^{m}$ is 1 .

(3) The coefficient of $\mathbf{i}^{m+1}$ in $\mathbf{i}^{m} \diamond \mathbf{i}$ is $(-1)^{p(m \mathbf{i})} q_{\mathbf{i}}^{-m}[m+1]_{\mathbf{i}}$.

Proof. Let $\mathbf{i} \succ \mathbf{j}$. We will prove that $\max \left(\mathbf{i}^{m} \diamond \mathbf{j}\right)=\mathbf{i}^{m} \mathbf{j}$ and (1) by induction on $m$. The case $m=1$ follows from [5, Lemma 4.5]. Assume that $\max \left(\mathbf{i}^{m-1} \diamond \mathbf{j}\right)=\mathbf{i}^{m-1} \mathbf{j}$ for all $\mathbf{j} \in \mathcal{W}^{+}$such that $\mathbf{i} \succ \mathbf{j}$. Suppose that the word $\mathbf{k}$ occurs as a nontrivial shuffle in $\mathbf{i}^{m} \diamond \mathbf{j}$ (i.e. $\mathbf{k} \neq \mathbf{i}^{m} \mathbf{j}$ ). Then there exists a factorization $\mathbf{j}=\mathbf{j}_{1} \mathbf{j}_{2}$ such 
that $\mathbf{k}$ occurs in $\left(\mathbf{i}^{m-1} \diamond \mathbf{j}_{1}\right)\left(\mathbf{i} \diamond \mathbf{j}_{2}\right)$. Clearly $\mathbf{i} \succ \mathbf{j} \succeq \mathbf{j}_{1}$ and $\mathbf{j}_{1} \in \mathcal{W}^{+}$(as a factor of a dominant word). Hence by the inductive assumption

$$
\mathbf{k} \preceq \mathbf{i}^{m-1} \mathbf{j}_{1} \max \left(\mathbf{i} \diamond \mathbf{j}_{2}\right)
$$

Now, since any word occurring in $\mathbf{j}_{1}\left(\mathbf{i} \diamond \mathbf{j}_{2}\right)$ is a proper shuffle in $\mathbf{i} \diamond\left(\mathbf{j}_{1} \mathbf{j}_{2}\right)=\mathbf{i} \diamond \mathbf{j}$ and the maximum word in $\mathbf{i} \diamond \mathbf{j}$ is $\mathbf{i j}$ we have

$$
\mathbf{k} \preceq \mathbf{i}^{m-1} \mathbf{j}_{1} \max \left(\mathbf{i} \diamond \mathbf{j}_{2}\right) \prec \mathbf{i}^{m-1} \max (\mathbf{i} \diamond \mathbf{j})=\mathbf{i}^{m} \mathbf{j}
$$

which proves that $\max \left(\mathbf{i}^{m} \diamond \mathbf{j}\right) \preceq \mathbf{i}^{m} \mathbf{j}$.

Next, we prove that the coefficient of $\mathbf{i}^{m} \mathbf{j}$ in $\mathbf{i}^{m} \diamond \mathbf{j}$ is $(-1)^{p(m \mathbf{i}) p(\mathbf{j})} q^{-(m|\mathbf{i}|,|\mathbf{j}|)}$ by induction on $\ell(\mathbf{j})$. Let $\mathbf{i}=\left(i_{1}, \ldots, i_{d}\right), \mathbf{j}=\left(j_{1}, \ldots, j_{k}\right)$ and assume that $\mathbf{i} \succ \mathbf{j}$. Suppose that $\ell(\mathbf{j})=1$, so $\mathbf{j}=j_{1}=j \in I$ and $\mathbf{i}>j$. Then we have $j<i_{1}$. We claim that the coefficient of $\mathbf{i}^{m} j$ in $\mathbf{i}^{m} \diamond j$ is $(-1)^{p(m \mathbf{i}) p(j)} q^{-\left(|m \mathbf{i}|, \alpha_{j}\right)}$. We have

$$
\begin{aligned}
\mathbf{i}^{m} \diamond j & =\mathbf{i}^{m-1}\left(i_{1}, \ldots, i_{d}\right) \diamond j \\
& =\left(\mathbf{i}^{m-1}\left(i_{1}, \ldots, i_{d-1}\right) \diamond j\right) i_{d}+(-1)^{p(m \mathbf{i}) p(j)} q^{-\left(|m \mathbf{i}|, \alpha_{j}\right)} \mathbf{i}^{m} j .
\end{aligned}
$$

We claim that $\max \left(\left(\mathbf{i}^{m-1}\left(i_{1}, \ldots, i_{d-1}\right) \diamond j\right) i_{d}\right) \prec \mathbf{i}^{m} j$. Indeed, $\mathbf{i}^{m-1}\left(i_{1}, \ldots, i_{d-1}\right) j i_{d} \prec \mathbf{i}^{m} j$ and any nontrivial shuffle in $\mathbf{i}^{m-1}\left(i_{1}, \ldots, i_{d-1}\right) \diamond j$ occurs as a shuffle in either $\left(\mathbf{i}^{m-1} \diamond j\right)\left(i_{1}, \ldots, i_{d-1}\right)$ or in $\mathbf{i}^{m-1}\left(\left(i_{1}, \ldots, i_{d-1}\right) \diamond j\right)$. By the above and Lemma 1.14 we have that $\max \left(\left(\mathbf{i}^{m-1} \diamond j\right)\left(i_{1}, \ldots, i_{d-1}\right)\right) i_{d} \prec \mathbf{i}^{m} j$ and $\max \left(\mathbf{i}^{m-1}\left(\left(i_{1}, \ldots, i_{d-1}\right) \diamond j\right)\right) i_{d} \prec \mathbf{i}^{m} j$. Therefore, $\max \left(\left(\mathbf{i}^{m-1}\left(i_{1}, \ldots, i_{d-1}\right) \diamond j\right) i_{d}\right) \prec \mathbf{i}^{m} j$ which proves that the coefficient of $\mathbf{i}^{m} j$ in $\mathbf{i}^{m} \diamond j$ has to be $(-1)^{p(m \mathbf{i}) p(j)} q^{-\left(|m \mathbf{i}|, \alpha_{j}\right)}$.

For the inductive step, assume that the coefficient of $\mathbf{i}^{m} \mathbf{w}$ is $(-1)^{p(m \mathbf{i}) p(\mathbf{w})} q^{-(m|\mathbf{i}|,|\mathbf{w}|)}$ for all $m$ and $\mathbf{w} \in \mathcal{W}^{+}$such that $\mathbf{i} \succ \mathbf{w}$ and $\ell(\mathbf{w})<\ell(\mathbf{j})$. We have

$$
\begin{aligned}
\mathbf{i}^{m} \diamond \mathbf{j} & =\mathbf{i}^{m-1}\left(i_{1}, \ldots, i_{d}\right) \diamond\left(j_{1}, \ldots, j_{k}\right) \\
& =\left(\mathbf{i}^{m-1}\left(i_{1}, \ldots, i_{d-1}\right) \diamond \mathbf{j}\right) i_{d}+(-1)^{p(m \mathbf{i}) p\left(j_{k}\right)} q^{-\left(|m \mathbf{i}|, \alpha_{j_{k}}\right)}\left(\mathbf{i}^{m} \diamond\left(j_{1}, \ldots, j_{k-1}\right)\right) j_{k} .
\end{aligned}
$$

Again we show that $\max \left(\left(\mathbf{i}^{m-1}\left(i_{1}, \ldots, i_{d-1}\right) \diamond \mathbf{j}\right) i_{d}\right) \prec \mathbf{i}^{m} \mathbf{j}$. Clearly, $\mathbf{i}^{m-1}\left(i_{1}, \ldots, i_{d-1}\right) \mathbf{j} i_{d} \prec \mathbf{i}^{m} \mathbf{j}$, and if $\mathbf{k}$ is any nontrivial shuffle in $\mathbf{i}^{m-1}\left(i_{1}, \ldots, i_{d-1}\right) \diamond \mathbf{j}$, then there exists a factorization $\mathbf{j}=\mathbf{j} \mathbf{j}_{1}$ such that $\mathbf{k}$ occurs in $\left(\mathbf{i}^{m-1} \diamond \mathbf{j}_{1}\right)\left(\left(i_{1}, \ldots, i_{d-1}\right) \diamond \mathbf{j}_{2}\right)$. Again $\mathbf{i} \succ \mathbf{j} \succeq \mathbf{j}_{1}$ and $\mathbf{j}_{1} \in \mathcal{W}^{+}$. Since $\max \left(\mathbf{i}^{m-1} \diamond \mathbf{j}_{1}\right)=\mathbf{i}^{m-1} \mathbf{j}_{1}$, it follows that

$$
\mathbf{k} \preceq \mathbf{i}^{m-1} \mathbf{j}_{1} \max \left(\left(i_{1}, \ldots, i_{d-1}\right) \diamond \mathbf{j}_{2}\right)
$$

Now, since any word occurring in $\mathbf{j}_{1}\left(\left(i_{1}, \ldots, i_{d-1}\right) \diamond \mathbf{j}_{2}\right)$ is a proper shuffle in $\left(i_{1}, \ldots, i_{d-1}\right) \diamond\left(\mathbf{j}_{1} \mathbf{j}_{2}\right)=$ $\left(i_{1}, \ldots, i_{d-1}\right) \diamond \mathbf{j}$, we have

$$
\mathbf{k} \prec \mathbf{i}^{m-1} \max \left(\left(i_{1}, \ldots, i_{d-1}\right) \diamond \mathbf{j}\right) \prec \mathbf{i}^{m-1} \max (\mathbf{i} \diamond \mathbf{j})=\mathbf{i}^{m} \mathbf{j}
$$

By induction on $\ell(\mathbf{j})$ and (1.16), it follows that the coefficient of $\mathbf{i}^{m} \mathbf{j}$ in $\mathbf{i}^{m} \diamond \mathbf{j}$ is $(-1)^{p(m \mathbf{i}) p(\mathbf{j})} q^{-(|m \mathbf{i}|,|\mathbf{j}|)}$, which proves (1).

Next, we prove that $\max \left(\mathbf{j} \diamond \mathbf{i}^{m}\right)=\mathbf{i}^{m} \mathbf{j}$. By Proposition 1.6, we have $\sigma(\mathbf{j})=(-1)^{P(\mathbf{j})} q^{-N(|\mathbf{j}|)} \mathbf{j}$ and $\sigma\left(\mathbf{i}^{m}\right)=(-1)^{P(m \mathbf{i})} q^{-N(m|\mathbf{i}|)} \mathbf{i}^{m}$. Since $\sigma$ is an anti-automorphism, we have

$$
\sigma\left(\mathbf{j} \diamond \mathbf{i}^{m}\right)=\sigma(\mathbf{i})^{m} \diamond \sigma(\mathbf{j})=(-1)^{P(m \mathbf{i})+P(\mathbf{j})} q^{-(N(m|\mathbf{i}|)+N(|\mathbf{j}|))} \mathbf{i}^{m} \diamond \mathbf{j}
$$


By Proposition 1.6, $\max (\sigma(u))=\max (u)$ for all $u \in \mathcal{U}$. Therefore by (1.17) we have $\max \left(\mathbf{j} \diamond \mathbf{i}^{m}\right)=$ $\max \left(\mathbf{i}^{m} \diamond \mathbf{j}\right)=\mathbf{i}^{m} \mathbf{j}$. Moreover, the coefficient of $\mathbf{i}^{m} \mathbf{j}$ in $\mathbf{i}^{m} \diamond \mathbf{j}$ is $(-1)^{p(m \mathbf{i}) p(\mathbf{j})} q^{-(m|\mathbf{i}|,|\mathbf{j}|)}$ by (1). Lemma 1.8 yields

$$
N(m|\mathbf{i}|)+N(|\mathbf{j}|)+(|m \mathbf{i}|,|\mathbf{j}|)=N\left(\left|\mathbf{i}^{m} \mathbf{j}\right|\right) \quad \text { and } \quad P(m \mathbf{i})+P(\mathbf{j})+p(m \mathbf{i}) p(\mathbf{j})=P\left(\mathbf{i}^{m} \mathbf{j}\right) .
$$

Consequently, for some coefficients $a_{\mathbf{k}} \in \mathcal{A}$, we have

$$
\sigma\left(\mathbf{j} \diamond \mathbf{i}^{m}\right)=(-1)^{P\left(\mathbf{i}^{m} \mathbf{j}\right)} q^{-N\left(\left|\mathbf{i}^{m} \mathbf{j}\right|\right)} \mathbf{i}^{m} \mathbf{j}+\sum_{\mathbf{k} \prec \mathbf{i}^{m} \mathbf{j}} a_{\mathbf{k}} \mathbf{k} .
$$

Since $\sigma^{2}=\operatorname{Id}{ }_{\mathcal{F}}, \sigma(q)=-q^{-1}, \sigma\left(\mathbf{i}^{m} \mathbf{j}\right)=(-1)^{P\left(\mathbf{i}^{m} \mathbf{j}\right)} q^{-N\left(\left|\mathbf{i}^{m} \mathbf{j}\right|\right)} \mathbf{i}^{m} \mathbf{j}$ and by Lemma 1.8(1) $N\left(\left|\mathbf{i}^{m} \mathbf{j}\right|\right)$ is even, it follows by (1.18) that

$$
\mathbf{j} \diamond \mathbf{i}^{m}=\mathbf{i}^{m} \mathbf{j}+\sum_{\sigma(\mathbf{k}) \prec \mathbf{i}^{m} \mathbf{j}} \sigma\left(a_{\mathbf{k}}\right) \sigma(\mathbf{k}) .
$$

This proves (2).

Next, we prove that $\max \left(\mathbf{i}^{m} \diamond \mathbf{i}\right)=\mathbf{i}^{m+1}$ and (3) by induction on $m$. Let $\mathbf{i}=\left(i_{1}, \ldots, i_{d}\right)$. Suppose that $m=1$. By [5, Lemma 4.5], $\max (\mathbf{i} \diamond \mathbf{i})=\mathbf{i}^{2}$ and the coefficient of $\mathbf{i}^{2}$ in $\mathbf{i} \diamond \mathbf{i}$ is

$$
1+(-1)^{p(\mathbf{i})} q^{-(|\mathbf{i}|,|\mathbf{i}|)}=1+(-1)^{p(\mathbf{i})} q_{\mathbf{i}}^{-2}=(-1)^{p(\mathbf{i})} q_{\mathbf{i}}^{-1}[2]_{\mathbf{i}}
$$

For the inductive step, assume that $\max \left(\mathbf{i}^{m-1} \diamond \mathbf{i}\right)=\mathbf{i}^{m}$ and that the coefficient of $\mathbf{i}^{m}$ in $\mathbf{i}^{m-1} \diamond \mathbf{i}$ is $(-1)^{(m-1) p(\mathbf{i})} q_{\mathbf{i}}^{-(m-1)}[m]_{\mathbf{i}}$. If $d=1$, then the result follows easily by induction on $m$ and the fact that

$$
(-1)^{(m-1) p(\mathbf{i})} q_{\mathbf{i}}^{-(m-1)}[m]_{\mathbf{i}}+(-1)^{p(m \mathbf{i}) p(\mathbf{i})} q^{-(m|\mathbf{i}|,|\mathbf{i}|)}=(-1)^{m p(\mathbf{i})} q_{\mathbf{i}}^{-m}[m+1]_{\mathbf{i}},
$$

which can be easily verified from (1.13). Assume that $d>1$ and let $\mathbf{j}=\left(i_{1}, \ldots, i_{d-1}\right)$. Then $\mathbf{j} \in \mathcal{W}^{+}$and $\mathbf{i} \succ \mathbf{j}$. Moreover,

$$
\mathbf{i}^{m} \diamond \mathbf{i}=\left(\mathbf{i}^{m-1} \mathbf{j} \diamond \mathbf{i}\right) i_{d}+(-1)^{p(m \mathbf{i}) p\left(i_{d}\right)} q^{-\left(m|\mathbf{i}|,\left|i_{d}\right|\right)}\left(\mathbf{i}^{m} \diamond \mathbf{j}\right) i_{d} .
$$

By part (2) there exist $b_{\mathbf{k}} \in \mathcal{A}$ such that

$$
\mathbf{i}^{m-1} \mathbf{j}=\mathbf{j} \diamond \mathbf{i}^{m-1}+\sum_{\mathbf{k} \prec \mathbf{i}^{m-1} \mathbf{j}} b_{\mathbf{k}} \mathbf{k}
$$

Hence, using (1.20), induction on $m,(1)$, and (2), we obtain for some $a_{\mathbf{h}}, c_{\mathbf{h}} \in \mathcal{A}$

$$
\begin{aligned}
\mathbf{i}^{m} \diamond \mathbf{i}= & \left(\left(\mathbf{j} \diamond \mathbf{i}^{m-1}\right) \diamond \mathbf{i}\right) i_{d}+\sum_{\mathbf{k} \prec \mathbf{i}^{m-1} \mathbf{j}} b_{\mathbf{k}}(\mathbf{k} \diamond \mathbf{i}) i_{d}+(-1)^{p(m \mathbf{i}) p\left(i_{d}\right)} q^{-\left(m|\mathbf{i}|,\left|i_{d}\right|\right)}\left(\mathbf{i}^{m} \diamond \mathbf{j}\right) i_{d} \\
= & \left(\mathbf{j} \diamond\left(\mathbf{i}^{m-1} \diamond \mathbf{i}\right)\right) i_{d}+(-1)^{p(m \mathbf{i}) p\left(i_{d}\right)} q^{-\left(m|\mathbf{i}|,\left|i_{d}\right|\right)}\left(\mathbf{i}^{m} \diamond \mathbf{j}\right) i_{d}+\sum_{\mathbf{k} \prec \mathbf{i}^{m-1} \mathbf{j}} b_{\mathbf{k}}(\mathbf{k} \diamond \mathbf{i}) i_{d} \\
= & (-1)^{(m-1) p(\mathbf{i})} q_{\mathbf{i}}^{-(m-1)}[m]_{\mathbf{i}}\left(\mathbf{j} \diamond \mathbf{i}^{m}\right) i_{d}+(-1)^{p(m \mathbf{i}) p\left(\left|i_{d}\right|+|\mathbf{j}|\right)} q^{-\left(m|\mathbf{i}|,\left|i_{d}\right|+|\mathbf{j}|\right)}\left(\mathbf{i}^{m} \mathbf{j}\right) i_{d} \\
& +\sum_{\mathbf{h} \prec \mathbf{i}^{m} \mathbf{j}} a_{\mathbf{h}} \mathbf{h} i_{d}+\sum_{\mathbf{k} \prec \mathbf{i}^{m-1} \mathbf{j}} b_{\mathbf{k}}(\mathbf{k} \diamond \mathbf{i}) i_{d} \\
= & \left((-1)^{p((m-1) \mathbf{i})} q_{\mathbf{i}}^{-(m-1)}[m]_{\mathbf{i}}+(-1)^{p(m \mathbf{i}) p(\mathbf{i})} q^{-(m|\mathbf{i}|,|\mathbf{i}|)}\right) \mathbf{i}^{m+1} \\
& +\sum_{\mathbf{h} \prec \mathbf{i}^{m} \mathbf{j}} c_{\mathbf{h}} \mathbf{h} i_{d}+\sum_{\mathbf{k} \prec \mathbf{i}^{m-1} \mathbf{j}} b_{\mathbf{k}}(\mathbf{k} \diamond \mathbf{i}) i_{d} .
\end{aligned}
$$


Moreover, $\mathbf{h} i_{d} \prec \mathbf{i}^{m} \mathbf{j} i_{d}=\mathbf{i}^{m+1}$ for all $\mathbf{h} \prec \mathbf{i}^{m} \mathbf{j}$ and by Lemma 1.14 we get that $\max (\mathbf{k} \diamond \mathbf{i}) i_{d} \prec$ $\max \left(\mathbf{i}^{m-1} \mathbf{j} \diamond \mathbf{i}\right) i_{d}=\left(\mathbf{i}^{m} \mathbf{j}\right) i_{d}=\mathbf{i}^{m+1}$ for all $\mathbf{k} \prec \mathbf{i}^{m-1} \mathbf{j}$. Hence $\max \left(\mathbf{i}^{m} \diamond \mathbf{i}\right)=\mathbf{i}^{m+1}$ and the leading coefficient is $(-1)^{m p(\mathbf{i})} q_{\mathbf{i}}^{-m}[m+1]_{\mathbf{i}}$ by (1.19).

Finally, $\max \left(\mathbf{i} \diamond \mathbf{i}^{m}\right)=\mathbf{i}^{m+1}$ since $\max (\sigma(u))=\max (u)$ for all $u \in \mathcal{U}$.

Corollary 1.21. Let $\mathbf{i} \in \mathcal{L}, \mathbf{j} \in \mathcal{W}^{+}, \mathbf{i} \succ \mathbf{j}$, and $m \in \mathbb{Z}_{>0}$. Then we have $\max \left(\mathbf{j} \diamond \mathbf{i}^{\diamond m}\right)=\mathbf{i}^{m} \mathbf{j}$ and the coefficient of $\mathbf{i}^{m} \mathbf{j}$ in $\mathbf{j} \diamond \mathbf{i}^{\diamond m}$ is $(-1)^{\frac{m(m-1)}{2}} p(\mathbf{i}) q_{\mathbf{i}}^{-\frac{m(m-1)}{2}}[m]_{\mathbf{i}}$ !.

Proof. First, we claim that $\max \left(\mathbf{i}^{\triangleright m}\right)=\mathbf{i}^{m}$ and that this top word appears with coefficient $(-1)^{\frac{m(m-1)}{2}} p(\mathbf{i}) q_{\mathbf{i}}^{-\frac{m(m-1)}{2}}[m]_{\mathbf{i}} !$. The case $m=1$ is trivial. Assume that $m>1$. Then by induction, Lemma 1.15(3), and Lemma 1.14, we have that for some $a_{\mathbf{h}} \in \mathcal{A}$,

$$
\begin{aligned}
\mathbf{i}^{\diamond m} & =\mathbf{i}^{\diamond(m-1)} \diamond \mathbf{i} \\
& =(-1)^{p(\mathbf{i})(m-1)(m-2) / 2} q_{\mathbf{i}}^{-(m-1)(m-2) / 2}[m-1]_{\mathbf{i}} !(-1)^{(m-1) p(\mathbf{i})} q_{\mathbf{i}}^{-(m-1)}[m]_{\mathbf{i}} \mathbf{i}^{m}+\sum_{\mathbf{h} \prec \mathbf{i}^{m}} a_{\mathbf{h}} \mathbf{h} \\
& =(-1)^{p(\mathbf{i}) m(m-1) / 2} q_{\mathbf{i}}^{-m(m-1) / 2}[m]_{\mathbf{i}} \mathbf{i}^{m}+\sum_{\mathbf{h} \prec \mathbf{i}^{m}} a_{\mathbf{h}} \mathbf{h} .
\end{aligned}
$$

Now the statement of the corollary follows from the above computation and Lemma 1.15(2).

Let $\mathbf{i} \in \mathcal{W}^{+}$with canonical factorization $\mathbf{i}=\mathbf{i}_{1}^{n_{1}} \ldots \mathbf{i}_{d}^{n_{d}}$, where $n_{1}, \ldots, n_{d} \in \mathbb{Z}_{>0}, \mathbf{i}_{1}, \ldots, \mathbf{i}_{d} \in \mathcal{L}^{+}$and $\mathbf{i}_{1} \succ \cdots \succ \mathbf{i}_{d}$. We define

$$
\xi(\mathbf{i})=\sum_{k=1}^{d} p\left(\mathbf{i}_{k}\right) n_{k}\left(n_{k}-1\right) / 2 \quad \text { and } \quad s(\mathbf{i})=\sum_{k=1}^{d}\left(\left|\mathbf{i}_{k}\right|,\left|\mathbf{i}_{k}\right|\right) n_{k}\left(n_{k}-1\right) / 4
$$

Corollary 1.23. With the notations above, we have, for some $a_{\mathbf{k}} \in \mathcal{A}$,

$$
\mathbf{i}_{d}^{\diamond n_{d}} \diamond \cdots \diamond \mathbf{i}_{1}^{\diamond n_{1}}=\left((-1)^{\xi(\mathbf{i})} q^{-s(\mathbf{i})} \prod_{k=1}^{d}\left[n_{k}\right]_{\mathbf{i}_{k}} !\right) \mathbf{i}+\sum_{\mathbf{k} \prec \mathbf{i}} a_{\mathbf{k}} \mathbf{k} .
$$

Proof. We will prove the statement by induction on $d$. If $d=1$, then $\mathbf{i}=\mathbf{i}_{1}^{n_{1}}$ and the result follows from Lemma 1.21 (1) since $\xi\left(\mathbf{i}_{1}^{n_{1}}\right)=p\left(\mathbf{i}_{1}\right) n_{1}\left(n_{1}-1\right) / 2$ and $q^{-s\left(\mathbf{i}_{1}^{n_{1}}\right)}=q^{-\left(\left|\mathbf{i}_{1}\right|,\left|\mathbf{i}_{1}\right|\right) n_{1}\left(n_{1}-1\right) / 4}=q_{\mathbf{i}_{1}}^{-n_{1}\left(n_{1}-1\right) / 2}$.

We now proceed to the inductive step. Suppose that $d>1$ and let $\mathbf{j}=\mathbf{i}_{2}^{n_{2}} \ldots \mathbf{i}_{d}^{n_{d}}$. Then $\mathbf{i}_{1} \succ \mathbf{j}$ and $\mathbf{j} \in \mathcal{W}^{+}$ by [5, Lemma 4.2]. By the inductive hypothesis we obtain

$$
\mathbf{i}_{d}^{\diamond n_{d}} \diamond \cdots \diamond \mathbf{i}_{2}^{\diamond n_{2}} \diamond \mathbf{i}_{1}^{\diamond n_{1}}=\left((-1)^{\xi(\mathbf{j})} q^{-s(\mathbf{j})} \prod_{k=2}^{d}\left[n_{k}\right]_{\mathbf{i}_{k}} !\right) \mathbf{j} \diamond \mathbf{i}_{1}^{n_{1}}+\sum_{\mathbf{h} \prec \mathbf{j}} b_{\mathbf{h}}\left(\mathbf{h} \diamond \mathbf{i}_{1}^{\diamond n_{1}}\right) .
$$

By Lemma 1.14, $\mathbf{h} \diamond \mathbf{i}_{1}^{\diamond n_{1}} \prec \mathbf{j} \diamond \mathbf{i}_{1}^{\diamond n_{1}}$ for all $\mathbf{h} \in \mathcal{W}$ such that $\mathbf{h} \prec \mathbf{j},|\mathbf{h}|=|\mathbf{j}|$. Moreover, by Lemma 1.21(2), $\max \left(\mathbf{j} \diamond \mathbf{i}^{\diamond n_{1}}\right)=\mathbf{i}_{1}^{n_{1}} \mathbf{j}=\mathbf{i}$ and the coefficient of $\mathbf{i}_{1}^{n_{1}} \mathbf{j}$ in $\mathbf{j} \diamond \mathbf{i}^{\diamond n_{1}}$ is

$$
(-1)^{p\left(\mathbf{i}_{1}\right) n_{1}\left(n_{1}-1\right) / 2} q_{\mathbf{i}_{1}}^{-n_{1}\left(n_{1}-1\right) / 2}\left[n_{1}\right]_{\mathbf{i}_{1}} !=(-1)^{\xi\left(\mathbf{i}_{1}^{n_{1}}\right)} q^{-s\left(\mathbf{i}_{1}^{n_{1}}\right)} .
$$

The statement of the corollary now follows from (1.24) and the equalities $\xi(\mathbf{i})=\xi\left(\mathbf{i}_{1}^{n_{1}}\right)+\xi(\mathbf{j})$ and $s(\mathbf{i})=$ $s\left(\mathbf{i}_{1}^{n_{1}}\right)+s(\mathbf{j})$. 


\section{5. $P B W$ and dual canonical bases}

For $1 \leq i \leq j \leq n$, we set

(1) $E_{\mathbf{i}}= \begin{cases}(-1)^{j-i}\left(q^{2}-q^{-2}\right)^{j-i} q^{-N(|\mathbf{i}|) \mathbf{i}} & \text { if } \mathbf{i}=(i, \ldots, j), \\ (-1)^{j-i}\left(q^{2}-q^{-2}\right)^{2 n-i-j} q^{-N(|\mathbf{i}|)}[2]_{n}^{-1} \mathbf{i} & \text { if } \mathbf{i}=(i, \ldots, n, n, \ldots, j), i<j ;\end{cases}$

(2) $E_{\mathbf{i}}^{*}= \begin{cases}\mathbf{i} & \text { if } \mathbf{i}=(i, \ldots, j), \\ {[2]_{n} \mathbf{i}} & \text { if } \mathbf{i}=(i, \ldots, n, n, \ldots, j), i<j .\end{cases}$

Let $\mathbf{i} \in \mathcal{W}^{+}$. As before, we write the canonical factorization of $\mathbf{i}$ in the form:

$$
\mathbf{i}=\mathbf{i}_{1}^{n_{1}} \cdots \mathbf{i}_{d}^{n_{d}}
$$

where $n_{1}, \ldots, n_{d} \in \mathbb{Z}_{>0}, \mathbf{i}_{1}, \ldots, \mathbf{i}_{d} \in \mathcal{L}^{+}$and $\mathbf{i}_{1} \succ \cdots \succ \mathbf{i}_{d}$. We define

$$
E_{\mathbf{i}}=E_{\mathbf{i}_{d}}^{\left(n_{d}\right)} \diamond \cdots \diamond E_{\mathbf{i}_{1}}^{\left(n_{1}\right)},
$$

where $E_{\mathbf{j}}^{(m)}=E_{\mathbf{j}}^{\diamond m} /[m]_{\mathbf{j}}$ ! for $\mathbf{j} \in \mathcal{L}^{+}$, and define

$$
E_{\mathbf{i}}^{*}=E_{\mathbf{i}} /\left(E_{\mathbf{i}}, E_{\mathbf{i}}\right),
$$

where $(\cdot, \cdot)$ is the nondegenerate bilinear form on $\mathcal{U}$ from Proposition 1.4. Explicit computations of the bilinear form can be found in [5, Theorem 5.7]. In particular, for $\mathbf{i}, \mathbf{j} \in \mathcal{W}^{+}$, we have $\left(E_{\mathbf{i}}, E_{\mathbf{j}}\right)=0$ unless $\mathbf{i}=\mathbf{j}$, and

$$
\left(E_{\mathbf{i}}, E_{\mathbf{i}}\right)=(-1)^{\xi(\mathbf{i})} q^{-s(\mathbf{i})} \prod_{k=1}^{d} \frac{\left(E_{\mathbf{i}_{k}}, E_{\mathbf{i}_{k}}\right)^{n_{k}}}{\left[n_{k}\right]_{\mathbf{i}_{k}} !}
$$

where $\xi(\mathbf{i})$ and $s(\mathbf{i})$ are defined in (1.22).

The sets $\left\{E_{\mathbf{i}} \mid \mathbf{i} \in \mathcal{W}^{+}\right\}$and $\left\{E_{\mathbf{i}}^{*} \mid \mathbf{i} \in \mathcal{W}^{+}\right\}$are bases for $\mathcal{U}$, called the $P B W$ basis and the dual $P B W$ basis, respectively.

Lemma 1.28. For $\mathbf{i} \in \mathcal{W}^{+}$with canonical factorization as in (1.25) we have

$$
E_{\mathbf{i}}^{*}=(-1)^{\xi(\mathbf{i})} q^{s(\mathbf{i})}\left(E_{\mathbf{i}_{d}}^{*}\right)^{\diamond n_{d}} \diamond \cdots \diamond\left(E_{\mathbf{i}_{1}}^{*}\right)^{\diamond n_{1}} .
$$

Proof. It follows from (1.26) and (1.27) that

$$
\begin{aligned}
& E_{\mathbf{i}}^{*}=\frac{E_{\mathbf{i}}}{\left(E_{\mathbf{i}}, E_{\mathbf{i}}\right)}=(-1)^{\xi(\mathbf{i})} q^{s(\mathbf{i})}\left(\prod_{k=1}^{d} \frac{\left[n_{k}\right]_{\mathbf{i}_{k}} !}{\left(E_{\mathbf{i}_{k}}, E_{\mathbf{i}_{k}}\right)^{n_{k}}}\right) E_{\mathbf{i}} \\
& =(-1)^{\xi(\mathbf{i})} q^{s(\mathbf{i})}\left(\prod_{k=1}^{d} \frac{\left[n_{k}\right]_{\mathbf{i}_{k}} !}{\left(E_{\mathbf{i}_{k}}, E_{\mathbf{i}_{k}}\right)^{n_{k}}}\right) E_{\mathbf{i}_{d}}^{\left(n_{d}\right)} \diamond \cdots \diamond E_{\mathbf{i}_{1}}^{\left(n_{1}\right)} \\
& =(-1)^{\xi(\mathbf{i})} q^{s(\mathbf{i})}\left(\prod_{k=1}^{d} \frac{\left[n_{k}\right]_{\mathbf{i}_{k}} !}{\left(E_{\mathbf{i}_{k}}, E_{\mathbf{i}_{k}}\right)^{n_{k}}}\right) \frac{E_{\mathbf{i}_{d}}^{\diamond n_{d}}}{\left[n_{d}\right]_{\mathbf{i}_{d}} !} \diamond \cdots \diamond \frac{E_{\mathbf{i}_{1}}^{\diamond n_{1}}}{\left[n_{1}\right]_{\mathbf{i}_{1}} !} \\
& =(-1)^{\xi(\mathbf{i})} q^{s(\mathbf{i})} \frac{E_{\mathbf{i}_{d}}^{\diamond n_{d}}}{\left(E_{\mathbf{i}_{d}}, E_{\mathbf{i}_{d}}\right)^{n_{d}}} \diamond \cdots \diamond \frac{E_{\mathbf{i}_{1}}^{\diamond n_{1}}}{\left(E_{\mathbf{i}_{1}}, E_{\mathbf{i}_{1}}\right)^{n_{1}}} \\
& =(-1)^{\xi(\mathbf{i})} q^{s(\mathbf{i})}\left(E_{\mathbf{i}_{d}}^{*}\right)^{\diamond n_{d}} \diamond \cdots \diamond\left(E_{\mathbf{i}_{1}}^{*}\right)^{\diamond n_{1}} \text {. }
\end{aligned}
$$


Define $\mathcal{U}_{\mathcal{A}}$ and $\mathcal{U}_{\mathcal{A}}^{*}$ to be the $\mathcal{A}$-subalgebras of $\mathcal{U}$ generated by $\left\{E_{\mathbf{i}} \mid \mathbf{i} \in \mathcal{W}^{+}\right\}$and $\left\{E_{\mathbf{i}}^{*} \mid \mathbf{i} \in \mathcal{W}^{+}\right\}$, respectively. Then we have

$$
\mathcal{U}_{\mathcal{A}}^{*}=\left\{v \in \mathcal{U} \mid(u, v) \in \mathcal{A} \text { for all } u \in \mathcal{U}_{\mathcal{A}}\right\}
$$

For $\mathbf{i}=\mathbf{i}_{1}^{n_{1}} \cdots \mathbf{i}_{d}^{n_{d}}$ as in $(1.25)$, set $\varsigma_{\mathbf{i}}=\left[n_{1}\right]_{\mathbf{i}_{1}} ! \cdots\left[n_{d}\right]_{\mathbf{i}_{d}}$ ! and consider the free $\mathcal{A}$-module $\mathcal{F}_{\mathcal{A}}=\bigoplus_{\mathbf{i} \in \mathcal{W}} \mathcal{A} \varsigma_{\mathbf{i}} \mathbf{i}$ Then we also have

$$
\mathcal{U}_{\mathcal{A}}^{*}=\mathcal{F}_{\mathcal{A}} \cap \mathcal{U},
$$

and $\mathcal{U}_{\mathcal{A}}^{*}$ is $Q^{+}$-graded with $\left(\mathcal{U}_{\mathcal{A}}^{*}\right)_{\alpha}=\mathcal{F}_{\mathcal{A}} \cap \mathcal{U}_{\alpha}$.

Theorem 1.30. ([5, Theorem \%.11]) There exists a basis $\left\{b_{\mathbf{i}}^{*} \mid \mathbf{i} \in \mathcal{W}^{+}\right\}$for $\mathcal{U}_{\mathcal{A}}^{*}$ characterized by the properties:

(1) $b_{\mathbf{i}}^{*}-E_{\mathbf{i}}^{*}$ is a linear combination of vectors $E_{\mathbf{j}}^{*}, \mathbf{j} \prec \mathbf{i}$, with coefficients $q \mathbb{Z}[q]$;

(2) If we write $b_{\mathbf{i}}^{*}=\sum_{\mathbf{j}} c_{\mathbf{j}} \mathbf{j}, c_{\mathbf{j}} \in \mathcal{A}$, then we have $\overline{c_{\mathbf{j}}}=c_{\mathbf{j}}$. (Recall $\bar{q}=-q^{-1}$.)

Furthermore, we have $\max \left(b_{\mathbf{i}}^{*}\right)=\mathbf{i}$ for all $\mathbf{i} \in \mathcal{W}^{+}$, and $b_{\mathbf{i}}^{*}=E_{\mathbf{i}}^{*}$ for $\mathbf{i} \in \mathcal{L}^{+}$.

The basis $B^{*}=\left\{b_{\mathbf{i}}^{*} \mid \mathbf{i} \in \mathcal{W}^{+}\right\}$is called the dual canonical basis for $\mathcal{U}_{\mathcal{A}}^{*}$. Let $\beta \in \Phi^{+}$and $m \in \mathbb{Z}_{\geq 0}$. Clearly, $E_{\boldsymbol{\iota}^{+}(\beta)^{m}}^{*} \in\left(U_{\mathcal{A}}^{*}\right)_{m \beta}$. Since $\left\{b_{\mathbf{i}}^{*}|| \mathbf{i} \mid=m \beta\right\}$ is a basis of $\left(\mathcal{U}_{\mathcal{A}}^{*}\right)_{m \beta}$ and by Corollary $1.12, \boldsymbol{\iota}^{+}(\beta)^{m}$ is the smallest dominant word in $\mathcal{W}_{m \beta}^{+}$, it follows from Theorem 1.30 that

Corollary 1.31. $E_{\iota+(\beta)^{m}}^{*}=b_{\iota+(\beta)^{m}}^{*}$.

\section{Spin quiver Hecke algebras}

\subsection{Generators and relations}

Let $\mathbb{K}$ be a field with char $\mathbb{K} \neq 2$, and let $\widetilde{\Gamma}$ be a quiver with compatible automorphism $a: \widetilde{\Gamma} \rightarrow \widetilde{\Gamma}$. Denote the set of vertices of $\widetilde{\Gamma}$ by $\widetilde{I}$ and the set of edges by $\widetilde{H}$. We have maps $s: \widetilde{H} \rightarrow \widetilde{I}$ and $t: \widetilde{H} \rightarrow \widetilde{I}$ such that $s(a(h))=a(s(h))$ and $t(a(h))=a(t(h))$ for all $h \in \widetilde{H}$. Set $I$ to be a set of representatives of the orbits of $\widetilde{I}$ under $a$ and let $\Gamma=\widetilde{\Gamma} / a$ be the Dynkin diagram with nodes labeled by $I$, assuming $\Gamma$ has no loops. For each $i \in I$, let $\alpha_{i} \in \widetilde{I} / a$ be the corresponding orbit. For $i \neq j$, we set

$$
\left(\alpha_{i}, \alpha_{i}\right)=2\left|\alpha_{i}\right| \quad \text { and } \quad\left(\alpha_{i}, \alpha_{j}\right)=-\left|\left\{\left(i^{\prime}, j^{\prime}\right) \in \widetilde{H} \mid i^{\prime} \in \alpha_{i}, j^{\prime} \in \alpha_{j}\right\}\right| .
$$

Then we obtain a generalized Cartan matrix $A=\left(a_{i j}\right)$ and a matrix $D=\operatorname{diag}\left(s_{1}, \ldots, s_{n}\right)$ by setting $s_{i}=\left|\alpha_{i}\right|$ and $a_{i j}=\left(\alpha_{i}, \alpha_{j}\right) / s_{i}$. Note that $D A$ is symmetric.

Now we assume that the matrix $A$ is the same as in (1.1), and put the same $\mathbb{Z} / 2 \mathbb{Z}$-grading on $I$, i.e. $I_{\overline{1}}=\{n\}$. The orbit $\alpha_{i}$ is to be identified with the simple root $\alpha_{i}$ of the Kac-Moody superalgebra $\mathfrak{g}$ associated to $A$, and we keep all the notations in the previous section.

Define $d_{i j}=\mid\left\{h \in \widetilde{H} \mid s(h) \in \alpha_{i}\right.$ and $\left.t(h) \in \alpha_{j}\right\} / a \mid$ for $i \neq j$. For $i, j \in I$, set

$$
\mathbb{K}_{i j}\{u, v\}=\mathbb{K}\langle u, v\rangle /\left\langle u v-(-1)^{p(i) p(j)} v u\right\rangle,
$$

and define $Q_{i i}(u, v)=0$ and

$$
Q_{i j}(u, v)=(-1)^{d_{i j}}\left(u^{2 / s_{i}}-v^{2 / s_{j}}\right) \in \mathbb{K}_{i j}\{u, v\} \quad \text { for } i \neq j
$$


Assume $\nu=\sum_{i \in I} c_{i} \alpha_{i} \in Q^{+}$with $\sum_{i \in I} c_{i}=d$. Set

$$
I^{\nu}=\left\{\mathbf{i}=\left(i_{1}, \ldots, i_{d}\right) \in I^{d} \mid \alpha_{i_{1}}+\cdots+\alpha_{i_{d}}=\nu\right\}
$$

The symmetric groups $S_{d}$ act on $I^{\nu}$ by place permutations; in particular, the transposition $s_{r}$ acts as

$$
s_{r} \cdot\left(i_{1}, \ldots, i_{r}, i_{r+1}, \ldots, i_{d}\right)=\left(i_{1}, \ldots, i_{r+1}, i_{r}, \ldots, i_{d}\right) .
$$

The $\mathbb{K}$-algebra $\mathcal{H}^{-}(\nu)$ with the identity $1_{\nu}$ is defined by the generators $e(\mathbf{i})\left(\mathbf{i} \in I^{\nu}\right), y_{r}(r=1, \ldots, d), \tau_{s}$ $(s=1, \ldots, d-1)$ satisfying the following relations:

$$
\begin{aligned}
& e(\mathbf{i}) e(\mathbf{j})=\delta_{\mathbf{i j}} e(\mathbf{i}) \text { for all } \mathbf{i}, \mathbf{j} \in I^{\nu} ; \quad \sum_{\mathbf{i} \in I^{\nu}} e(\mathbf{i})=1_{\nu} ; \\
& y_{r} e(\mathbf{i})=e(\mathbf{i}) y_{r} \\
& \tau_{r} e(\mathbf{i})=e\left(s_{r} \cdot \mathbf{i}\right) \tau_{r} \\
& y_{r} y_{s} e(\mathbf{i})=(-1)^{p\left(i_{r}\right) p\left(i_{s}\right)} y_{s} y_{r} e(\mathbf{i}) \text { for } r \neq s \text {; } \\
& \tau_{r} y_{s} e(\mathbf{i})=(-1)^{p\left(i_{r}\right) p\left(i_{r+1}\right) p\left(i_{s}\right)} y_{s} \tau_{r} e(\mathbf{i}) \text { for } s \neq r, r+1 \text {; } \\
& \tau_{r} \tau_{s} e(\mathbf{i})=(-1)^{p\left(i_{r}\right) p\left(i_{r+1}\right) p\left(i_{s}\right) p\left(i_{s+1}\right)} \tau_{s} \tau_{r} e(\mathbf{i}) \text { for }|s-r|>1 \text {; } \\
& \tau_{r} y_{r+1} e(\mathbf{i})= \begin{cases}\left((-1)^{p\left(i_{r}\right) p\left(i_{r+1}\right)} y_{r} \tau_{r}+1\right) e(\mathbf{i}) & \text { if } i_{r}=i_{r+1}, \\
(-1)^{p\left(i_{r}\right) p\left(i_{r+1}\right)} y_{r} \tau_{r} e(\mathbf{i}) & \text { if } i_{r} \neq i_{r+1}\end{cases} \\
& y_{r+1} \tau_{r} e(\mathbf{i})= \begin{cases}\left((-1)^{p\left(i_{r}\right) p\left(i_{r+1}\right)} \tau_{r} y_{r}+1\right) e(\mathbf{i}) & \text { if } i_{r}=i_{r+1}, \\
(-1)^{p\left(i_{r}\right) p\left(i_{r+1}\right)} \tau_{r} y_{r} e(\mathbf{i}) & \text { if } i_{r} \neq i_{r+1}\end{cases} \\
& \tau_{r}^{2} e(\mathbf{i})=Q_{i_{r}, i_{r+1}}\left(y_{r}, y_{r+1}\right) e(\mathbf{i}) ; \\
& \left(\tau_{r} \tau_{r+1} \tau_{r}-\tau_{r+1} \tau_{r} \tau_{r+1}\right) e(\mathbf{i}) \\
& = \begin{cases}\left(\frac{Q_{i_{r}, i_{r+1}}\left(y_{r+2}, y_{r+1}\right)-Q_{i_{r}, i_{r+1}}\left(y_{r}, y_{r+1}\right)}{y_{r+2}-y_{r}}\right) e(\mathbf{i}) & \text { if } i_{r}=i_{r+2} \neq n, \\
(-1)^{p\left(i_{r+1}\right)}\left(y_{r+2}-y_{r}\right)\left(\frac{Q_{i_{r}, i_{r+1}}\left(y_{r+2}, y_{r+1}\right)-Q_{i_{r}, i_{r+1}}\left(y_{r}, y_{r+1}\right)}{y_{r+2}^{2}-y_{r}^{2}}\right) e(\mathbf{i}) & \text { if } i_{r}=i_{r+2}=n, \\
0 & \text { otherwise. }\end{cases}
\end{aligned}
$$

Now the spin quiver Hecke algebra is defined to be $\mathcal{H}^{-}=\bigoplus_{\nu \in Q^{+}} \mathcal{H}^{-}(\nu)$. We define a $\mathbb{Z}$-grading on $\mathcal{H}^{-}$ by $\operatorname{deg} e(\mathbf{i})=0, \operatorname{deg} y_{r} e(\mathbf{i})=\left(\alpha_{i_{r}}, \alpha_{i_{r}}\right)$ and $\operatorname{deg} \tau_{r} e(\mathbf{i})=-\left(\alpha_{i_{r}}, \alpha_{i_{r+1}}\right)$, and a $\mathbb{Z} / 2 \mathbb{Z}$-grading by $p(e(\mathbf{i}))=\overline{0}$, $p\left(y_{r} e(\mathbf{i})\right)=p\left(i_{r}\right)$ and $p\left(\tau_{r} e(\mathbf{i})\right)=p\left(i_{r}\right) p\left(i_{r+1}\right)$.

\subsection{Module categories}

Let $\operatorname{Mod}^{-}(\nu)$ be the abelian category of finitely generated $(\mathbb{Z} \times \mathbb{Z} / 2 \mathbb{Z})$-graded left $\mathcal{H}^{-}(\nu)$-modules. We write $\operatorname{Hom}_{\nu}$ for $\operatorname{Hom}_{\mathcal{H}^{-}(\nu)}$. For any $M \in \operatorname{Mod}^{-}(\nu)$, define its $q$-superdimension by

$$
\operatorname{dim}_{q}^{-} M=\sum_{k \in \mathbb{Z}}\left(\operatorname{dim} M_{\overline{0}}[k]-\operatorname{dim} M_{\overline{1}}[k]\right) q^{k} \in \mathbb{Z}((q)),
$$

and define the graded character by

$$
\operatorname{ch}_{q}^{-} M=\sum_{\mathbf{i} \in I^{\nu}}\left(\operatorname{dim}_{q}^{-} e(\mathbf{i}) M\right) \mathbf{i}
$$


The parity shift functor $\Pi: \operatorname{Mod}^{-}(\nu) \rightarrow \operatorname{Mod}^{-}(\nu)$ is defined by $(\Pi M)_{\overline{0}}=M_{\overline{1}}$ and $(\Pi M)_{\overline{1}}=M_{\overline{0}}$. We denote by $M\{m\}$ the same $\mathcal{H}^{-}(\nu)$-module $M$ with the $\mathbb{Z}$-grading shifted by $m \in \mathbb{Z}$, i.e. $M\{m\}[k]=M[k-m]$ for $k \in \mathbb{Z}$. Then the grading shift functor $q: \operatorname{Mod}^{-}(\nu) \rightarrow \operatorname{Mod}^{-}(\nu)$ is defined by $q M=M\{1\}$.

Set $\operatorname{Hom}_{\nu}^{-}(M, N)=\operatorname{Hom}_{\nu}(M, N) \oplus \operatorname{Hom}_{\nu}(M, \Pi N)$ with $\mathbb{Z} / 2 \mathbb{Z}$-grading given by

$$
\operatorname{Hom}_{\nu}^{-}(M, N)_{\overline{0}}=\operatorname{Hom}_{\nu}(M, N) \text { and } \operatorname{Hom}_{\nu}^{-}(M, N)_{\overline{1}}=\operatorname{Hom}_{\nu}(M, \Pi N),
$$

and define

$$
\operatorname{HOM}_{\nu}^{-}(M, N)=\bigoplus_{m \in \mathbb{Z}} \operatorname{Hom}_{\nu}^{-}\left(M, \Pi^{m} N\{m\}\right)
$$

Let $\mathcal{A}=\mathbb{Z}\left[q, q^{-1}\right]$ as before. The full subcategory of $\operatorname{Mod}^{-}(\nu)$ consisting of finite dimensional (resp. finitely generated projective) modules is denoted by $\operatorname{Rep}^{-}(\nu)$ (resp. $\operatorname{Proj}^{-}(\nu)$ ), and the corresponding Grothendieck group by $\left[\operatorname{Rep}^{-}(\nu)\right]$ (resp. $\left.\left[\operatorname{Proj}^{-}(\nu)\right]\right)$. The functors $\Pi$ and $q$ define $\mathcal{A}$-module structures on both $\operatorname{Rep}^{-}(\nu)$ and $\operatorname{Proj}^{-}(\nu)$ via $q[M]=[q M]$ and $-[M]=[\Pi M]$.

There is a unique $\mathbb{K}$-linear anti-automorphism $\psi: \mathcal{H}^{-}(\nu) \rightarrow \mathcal{H}^{-}(\nu)$ defined by $\psi(\mathbf{i})=\mathbf{i}, \psi\left(y_{r}\right)=y_{r}$ and $\psi\left(\tau_{s}\right)=\tau_{s}$ for all $\mathbf{i} \in I^{\nu}$ and $1 \leq r \leq d, 1 \leq s<d$. For a graded right $\mathcal{H}^{-}(\nu)$-module $M$, we define $M^{\psi}$ to be the left module with the action given by $x . m=m . \psi(x)$ for $m \in M$ and $x \in \mathcal{H}^{-}(\nu)$. Similarly, for a graded left $\mathcal{H}^{-}(\nu)$-module, we denote by the same notation $M^{\psi}$ the right module with the action twisted by $\psi$. Define $P^{\#}=\operatorname{HOM}_{\nu}^{-}\left(P, \mathcal{H}^{-}(\nu)\right)^{\psi}$ for $P \in \operatorname{Proj}^{-}(\nu)$, and the $\mathbb{Z}$-linear bar-involution on $\left[\operatorname{Proj}^{-}(\nu)\right]$ by $\bar{q}=-q^{-1}$ and $\overline{[P]}=\left[P^{\#}\right]$. We define a bilinear form $(\cdot, \cdot):\left[\operatorname{Proj}^{-}(\nu)\right] \times\left[\operatorname{Proj}^{-}(\nu)\right] \rightarrow \mathbb{Z}((q))$ by

$$
([P],[Q])=\operatorname{dim}_{q}^{-}\left(P^{\psi} \otimes_{\mathcal{H}^{-}} Q\right)=\operatorname{dim}_{q}^{-} \operatorname{HOM}_{\nu}^{-}\left(P^{\#}, Q\right) .
$$

For $M \in \operatorname{Rep}^{-}(\nu)$, we define its graded dual $M^{\circledast}=\operatorname{HOM}_{\mathbb{K}}^{-}(M, \mathbb{K})^{\psi}$ with the $\mathcal{H}^{-}(\nu)$-action given by $(x . f)(m)=f(\psi(x) . m)$ for $x \in \mathcal{H}^{-}(\nu), f \in M^{\circledast}$ and $m \in M$, where we set $\mathbb{K}=\mathbb{K}_{\overline{0}}$. Then we obtain $M^{\circledast} \in \operatorname{Rep}^{-}(\nu)$. A bar-involution on $\left[\operatorname{Rep}^{-}(\nu)\right]$ is defined by $\bar{q}=-q^{-1}$ and $\overline{[M]}=\left[M^{\circledast}\right]$. Define an $\mathcal{A}$-pairing $\langle\cdot, \cdot\rangle:\left[\operatorname{Proj}^{-}(\nu)\right] \times\left[\operatorname{Rep}^{-}(\nu)\right] \longrightarrow \mathcal{A}$ by

$$
\langle[P],[M]\rangle=\operatorname{dim}_{q}^{-} \operatorname{HOM}_{\nu}^{-}\left(P^{\#}, M\right) .
$$

For each irreducible representation $L \in \operatorname{Rep}^{-}(\nu)$, there exists a projective indecomposable cover $P_{L} \in$ $\operatorname{Proj}^{-}(\nu)$, which is dual to $L$ with respect to the pairing. Every element of $\operatorname{Proj}^{-}(\nu)$ is a direct sum of indecomposable representations of the form $P_{L}\{m\}$ for some irreducible $L$ and $m \in \mathbb{Z}$. Thus the pairing $\langle\cdot, \cdot\rangle$ is a perfect pairing.

\subsection{Induction and restriction}

Let $\mu, \nu \in Q^{+}$, and set $1_{\mu, \nu}=\sum_{\mathbf{i} \in I^{\mu}, \mathbf{j} \in I^{\nu}} e(\mathbf{i j})$. We have the natural embedding $\mathcal{H}^{-}(\mu) \otimes \mathcal{H}^{-}(\nu) \hookrightarrow$ $\mathcal{H}^{-}(\mu+\nu)$. Define the functor $\operatorname{Res}_{\mu, \nu}^{\mu+\nu}: \operatorname{Mod}^{-}(\mu+\nu) \rightarrow \operatorname{Mod}^{-}(\mu) \otimes \operatorname{Mod}^{-}(\nu)$ by $\operatorname{Res}_{\mu, \nu}^{\mu+\nu} M=1_{\mu, \nu} M$, and the functor $\operatorname{Ind}_{\mu, \nu}^{\mu+\nu}: \operatorname{Mod}^{-}(\mu) \otimes \operatorname{Mod}^{-}(\nu) \rightarrow \operatorname{Mod}^{-}(\mu+\nu)$ by

$$
\operatorname{Ind}_{\mu, \nu}^{\mu+\nu}(M \otimes N)=\mathcal{H}^{-}(\mu+\nu) 1_{\mu, \nu} \bigotimes_{\mathcal{H}^{-}(\mu) \otimes \mathcal{H}^{-}(\nu)}(M \otimes N) .
$$

Then we obtain the functors

$$
\text { Ind }=\bigoplus_{\mu, \nu} \operatorname{Ind}_{\mu, \nu}^{\mu+\nu} \quad \text { and } \quad \operatorname{Res}=\bigoplus_{\substack{\lambda, \mu, \nu \\ \mu+\nu=\lambda}} \operatorname{Res}_{\mu, \nu}^{\lambda} .
$$


Set $\left[\operatorname{Proj}^{-}\right]=\bigoplus_{\nu \in Q^{+}}\left[\operatorname{Proj}^{-}(\nu)\right]$ and $\left[\operatorname{Rep}^{-}\right]=\bigoplus_{\nu \in Q^{+}}\left[\operatorname{Rep}^{-}(\nu)\right]$. Then [Ind] defines a multiplication on $\left[\mathrm{Proj}^{-}\right]$to make it an $\mathcal{A}$-algebra. Similarly, [Rep ${ }^{-}$] becomes an $\mathcal{A}$-algebra with [Ind]. Furthermore, [Res] defines a comultiplication on both $\left[\mathrm{Proj}^{-}\right]$and $\left[\mathrm{Rep}^{-}\right]$to make them $\mathcal{A}$-coalgebras.

\subsection{Categorification of $\mathcal{U}_{\mathcal{A}}$ and $\mathcal{U}_{\mathcal{A}}^{*}$}

Theorem 2.11. ([r,9]) There exists a $\mathbb{Z} \times \mathbb{Z} / 2 \mathbb{Z}$-graded $\mathcal{A}$-algebra isomorphism $\gamma: \mathcal{U}_{\mathcal{A}} \stackrel{\sim}{\longrightarrow}\left[\operatorname{Proj}^{-}\right]$commuting with the bar-involutions on $\mathcal{U}_{\mathcal{A}}$ and $\left[\operatorname{Proj}^{-}\right]$.

Corollary 2.12. The induced map $\gamma^{*}:\left[\operatorname{Rep}^{-}\right] \stackrel{\sim}{\rightarrow} \mathcal{U}_{\mathcal{A}}^{*}$ is an isomorphism of $\mathbb{Z} \times \mathbb{Z} / 2 \mathbb{Z}$-graded $\mathcal{A}$-algebras.

For $M \in \operatorname{Mod}^{-}(\mu)$ and $N \in \operatorname{Mod}^{-}(\nu)$, we define

$$
M \circ N:=\operatorname{Ind}_{\mu, \nu}^{\mu+\nu}(M \otimes N)
$$

Proposition 2.13. Let $\mu, \nu \in Q^{+}, M \in \operatorname{Rep}^{-}(\mu)$ and $N \in \operatorname{Rep}^{-}(\nu)$. Then we have

$$
\operatorname{ch}_{q}^{-}(M \circ N)=\operatorname{ch}_{q}^{-}(N) \diamond \operatorname{ch}_{q}^{-}(M) .
$$

Proof. Choose a homogeneous basis $\left\{v_{1}, \ldots, v_{k}\right\}$ for $e(\mathbf{i}) M$ and $\left\{u_{1}, \ldots, u_{l}\right\}$ for $e(\mathbf{j}) N$. Then we obtain a basis $\left\{\tau_{w} v_{p} \otimes u_{q}: 1 \leq p \leq k, 1 \leq q \leq l, w \in S_{a+b} / S_{a} \times S_{b}\right\}$ of the homogeneous space of $M \circ N$. One can see that we need only to prove

$$
\sum_{w \in S_{a+b} / S_{a} \times S_{b}} c\left(\tau_{w}\right) w(\mathbf{i j})=\mathbf{j} \diamond \mathbf{i}
$$

where $c\left(\tau_{w}\right)=(-1)^{p\left(\tau_{w} e(\mathbf{i j})\right)} q^{\operatorname{deg}\left(\tau_{w} e(\mathbf{i j})\right)}$.

We use the inductive formula (1.2). Consider $\mathbf{i}=\left(i_{1}, \ldots, i_{a}\right)$ and $\mathbf{j}=\left(i_{a+1}, \ldots, i_{a+b}\right)$. Then we have

$$
\begin{aligned}
& \sum_{w \in S_{a+b} / S_{a} \times S_{b}} c\left(\tau_{w}\right) w(\mathbf{i j}) \\
& =\left(\sum_{w \in S_{(a-1)+b} / S_{(a-1)} \times S_{b}} c\left(\tau_{w}\right)\right) c\left(\tau_{a+b-1} \cdots \tau_{a+1} \tau_{a}\right) w \tau_{a+b-1} \cdots \tau_{a+1} \tau_{a}(\mathbf{i j}) \\
& \quad+\sum_{w \in S_{a+(b-1)} / S_{a} \times S_{(b-1)}} c\left(\tau_{w}\right) w\left(\mathbf{i}\left(i_{a+1}, \ldots, i_{a+b-1}\right)\right) i_{a+b} .
\end{aligned}
$$

On the other hand, we have

$$
\begin{aligned}
\mathbf{j} \diamond \mathbf{i}= & \left(i_{a+1}, \ldots, i_{a+b}\right) \diamond\left(i_{1}, \ldots, i_{a}\right) \\
= & (-1)^{p\left(x i_{a+b}\right) p\left(i_{a}\right)} q^{-\left(\left|x i_{a+b}\right|,\left|i_{a}\right|\right)}\left(\left(i_{a+1}, \ldots, i_{a+b}\right) \diamond\left(i_{1}, \ldots, i_{a-1}\right)\right) i_{a} \\
& \quad+\left(\left(i_{a+1}, \ldots, i_{a+b-1}\right) \diamond\left(i_{1}, \ldots, i_{a}\right)\right) i_{a+b},
\end{aligned}
$$

where $x=\left(i_{a+1}, \ldots, i_{a+b-1}\right)$. Since $c\left(\tau_{a+b-1} \cdots \tau_{a+1} \tau_{a}\right)=(-1)^{p\left(x i_{a+b}\right) p\left(i_{a}\right)} q^{-\left(\left|x i_{a+b}\right|,\left|i_{a}\right|\right)}$, we are done by induction.

We have the following important property of the map $\mathrm{ch}_{q}^{-}$, which is proved in [9, Corollary 8.16].

Proposition 2.14. ([9]) Let $M, M^{\prime} \in \operatorname{Rep}^{-}(\mu)$. If $\operatorname{ch}_{q}^{-}(M)=\operatorname{ch}_{q}^{-}\left(M^{\prime}\right)$, then $[M]=\left[M^{\prime}\right]$. 


\section{Cuspidal representations}

In this section we give an explicit construction of the cuspidal modules with the ordering we fixed on $I$ : $1 \prec 2 \prec \cdots \prec n$. These cuspidal modules will be building blocks for irreducible modules. We begin with the definition of a cuspidal module.

\section{Definition 3.1.}

(1) Let $\nu \in Q^{+}$and $M \in \operatorname{Rep}^{-}(\nu)$. The word $\mathbf{i}=\max \left(\operatorname{ch}_{q}^{-} M\right)$ is called the highest weight of $M$.

(2) Let $\alpha \in \Phi^{+}$. An irreducible $\mathcal{H}^{-}(\alpha)$-module $L$ is called cuspidal if the highest weight of $L$ is a dominant Lyndon word.

The set $\Phi^{+}$of reduced positive roots is

$$
\Phi^{+}=\{\alpha(i, j) \mid 1 \leq i \leq j \leq n\} \cup\{\beta(i, j) \mid 1 \leq i<j \leq n\},
$$

where $\alpha(i, j):=\sum_{r=i}^{j} \alpha_{r}$ and $\beta(i, j):=\sum_{r=i}^{j-1} \alpha_{r}+\sum_{r=j}^{n} 2 \alpha_{r}$. It follows from Proposition 1.10 that the corresponding dominant Lyndon words are:

$$
\boldsymbol{\iota}^{+}(\alpha(i, j))=(i, \ldots, j), \quad 1 \leq i \leq j \leq n, \quad \iota^{+}(\beta(i, j))=(i, \ldots, n, n, \ldots j), \quad 1 \leq i<j \leq n .
$$

The corresponding dual canonical bases elements are

$$
\begin{array}{ll}
E_{\iota^{+}(\alpha(i, j))}^{*}=(i, \ldots, j), & 1 \leq i \leq j \leq n, \\
E_{\iota^{+}(\beta(i, j))}^{*}=\left(-q+q^{-1}\right)(i, \ldots, n, n, \ldots, j), & 1 \leq i<j \leq n .
\end{array}
$$

Proposition 3.2. Let $\alpha \in \Phi^{+}$. For $\alpha=\alpha(i, j)$, we have the corresponding 1-dimensional cuspidal module $L_{\alpha}=\mathbb{K} v_{\alpha}$ with the action of the generators:

$$
e(\mathbf{j}) v_{\alpha}=\delta_{\mathbf{j}, \iota^{+}(\alpha)} v_{\alpha}, \quad \tau_{r} v_{\alpha}=y_{r} v_{\alpha}=0 \text { for all } r .
$$

For $\alpha=\beta(i, j), i<j$, we have the 2-dimensional cuspidal module $L_{\alpha}:=\mathbb{K} v_{1} \oplus \mathbb{K} v_{-1}$, where $\operatorname{deg} v_{g}=g$ for $g= \pm 1$, and $p\left(v_{1}\right)=\overline{1}, p\left(v_{-1}\right)=\overline{0}$, and the action of generators are given by:

$$
\begin{array}{ll}
e(\mathbf{j}) v_{g}=\delta_{\mathbf{j}, \iota^{+}(\alpha)} v_{g} & \text { for } g= \pm 1 ; \\
y_{r} v_{1}=0 & \text { for all } r ; \\
y_{r} v_{-1}=0 & \text { if } r \neq n-i+1, n-i+2 ; \\
y_{r} v_{-1}=v_{1} & \text { if } r=n-i+1, n-i+2 ; \\
\tau_{r} v_{1}=0 & \text { if } r \neq n-i+1 ; \\
\tau_{n-i+1} v_{1}=v_{-1} ; & \\
\tau_{r} v_{-1}=0 & \text { for all } r .
\end{array}
$$

Proof. If $\alpha=\alpha(i, j)$, then it is straightforward to check that the action satisfies (2.1)-(2.10). We clearly have $\operatorname{ch}_{q}^{-}\left(L_{\alpha}\right)=\iota^{+}(\alpha)=(i, \ldots, j)$. Thus $L_{\alpha}$ is a cuspidal representation.

Assume that $\alpha=\beta(i, j), i<j$, and consider the action of generators on $L_{\alpha}=\mathbb{K} v_{1} \oplus \mathbb{K} v_{-1}$. Clearly the relation (2.1) holds. For $g= \pm 1$, notice that

$$
\begin{aligned}
& y_{r} e(\mathbf{i}) v_{g}=y_{r} v_{g}=e(\mathbf{i}) y_{r} v_{g}, \quad \text { if } \mathbf{i}=\boldsymbol{\iota}^{+}(\alpha) \text {; } \\
& y_{r} e(\mathbf{i}) v_{g}=0=e(\mathbf{i}) y_{r} v_{g} \quad \text { otherwise. }
\end{aligned}
$$

Thus the relation (2.2) holds. 
We have

$$
\tau_{r} e(\mathbf{i}) v_{g}= \begin{cases}v_{-1} & \text { if } \mathbf{i}=\iota^{+}(\alpha), r=n-i+1, g=1 \\ 0 & \text { otherwise }\end{cases}
$$

Since $e\left(s_{r} \cdot \mathbf{i}\right)=e(\mathbf{i})$ for $r=n-i+1$, we get

$$
\begin{aligned}
e\left(s_{r} \cdot \mathbf{i}\right) \tau_{r} v_{g} & = \begin{cases}e\left(s_{r} \cdot \mathbf{i}\right) v_{-1} & \text { if } r=n-i+1, g=1, \\
0 & \text { otherwise },\end{cases} \\
& = \begin{cases}v_{-1} & \text { if } \mathbf{i}=\iota^{+}(\alpha), r=n-i+1, g=1, \\
0 & \text { otherwise }\end{cases}
\end{aligned}
$$

Thus $\tau_{r} e(\mathbf{i}) v_{g}=e\left(s_{r} \cdot \mathbf{i}\right) \tau_{r} v_{g}$ for $g= \pm 1$, and the relation (2.3) holds.

For the relations (2.4)-(2.10), we may assume that $\mathbf{i}=\boldsymbol{\iota}^{+}(\alpha)$ and will drop $e(\mathbf{i})$ from consideration. Since $y_{r} y_{s} v_{g}=0$ for any $r, s$ and $g= \pm 1$, the relation (2.4) is valid. For the relation (2.5), we assume that $s \neq r, r+1$. Then

$$
\tau_{r} y_{s} v_{g}= \begin{cases}\tau_{r} v_{1} & \text { if } s=n-i+1, n-i+2, g=-1 \\ 0 & \text { otherwise }\end{cases}
$$

Since $r \neq n-i+1$ from the assumption, we obtain $\tau_{r} y_{s} v_{g}=0$. Similarly, $y_{s} \tau_{r} v_{g}=0$, and the relation (2.5) holds. Next we have $\tau_{r} \tau_{s} v_{g}=0$ for any $r, s$, and the relation (2.6) is valid.

Now we see

$$
\begin{aligned}
\tau_{r} y_{r+1} v_{g} & = \begin{cases}\tau_{r} v_{1} & \text { if } r=n-i, n-i+1, g=-1, \\
0 & \text { otherwise }\end{cases} \\
& = \begin{cases}v_{-1} & \text { if } r=n-i+1, g=-1, \\
0 & \text { otherwise }\end{cases}
\end{aligned}
$$

On the other hand, if $r=n-i+1, g=-1$, then $i_{r}=i_{r+1}=n$ and

$$
\left((-1)^{p\left(i_{r}\right) p\left(i_{r+1}\right)} y_{r} \tau_{r}+1\right) v_{g}=\left(-y_{r} \tau_{r}+1\right) v_{-1}=v_{-1} .
$$

If $r=n-i+1, g=1$, then

$$
\left((-1)^{p\left(i_{r}\right) p\left(i_{r+1}\right)} y_{r} \tau_{r}+1\right) v_{g}=\left(-y_{r} \tau_{r}+1\right) v_{1}=-y_{r} v_{-1}+v_{1}=-v_{1}+v_{1}=0 .
$$

If $r \neq n-i+1$ then $i_{r} \neq i_{r+1}$ and $(-1)^{p\left(i_{r}\right) p\left(i_{r+1}\right)} y_{r} \tau_{r} v_{g}=0$. Consequently, the relation (2.7) holds. The relation (2.8) can be verified similarly, and we omit the details.

Clearly, $\tau_{r}^{2} v_{g}=0$ for any $r$ and $g= \pm 1$. On the other hand, if $r \neq n-i, n-i+2$ then we obtain immediately $Q_{i_{r}, i_{r+1}}\left(y_{r}, y_{r+1}\right) v_{g}=0$. If $r=n-i$ then

$$
Q_{i_{r}, i_{r+1}}\left(y_{r}, y_{r+1}\right) v_{g}=Q_{n-1, n}\left(y_{r}, y_{r+1}\right) v_{g}= \pm\left(y_{r}-y_{r+1}^{2}\right) v_{g}=0 .
$$

Similarly, if $r=n-i+2$ then

$$
Q_{i_{r}, i_{r+1}}\left(y_{r}, y_{r+1}\right) v_{g}=Q_{n, n-1}\left(y_{r}, y_{r+1}\right) v_{g}= \pm\left(y_{r}^{2}-y_{r+1}\right) v_{g}=0 .
$$


Thus we see that the relation (2.9) holds. Finally, $\left(\tau_{r} \tau_{r+1} \tau_{r}-\tau_{r+1} \tau_{r} \tau_{r+1}\right) v_{g}=0$ for any $r$ and $g= \pm 1$, while $i_{r} \neq i_{r+2}$ for any $r$. Hence it is easy to see that the relation (2.10) is valid.

Now we have shown that all the relations (2.1)-(2.10) are compatible with the action of the generators on the module $L_{\alpha}$, making it indeed an $\mathcal{H}^{-}(\alpha)$-module. Furthermore,

$$
\operatorname{ch}_{q}^{-}\left(L_{\alpha}\right)=\left(-q+q^{-1}\right) \iota^{+}(\alpha)=\left(-q+q^{-1}\right)(i, \ldots, n, n \ldots, j) .
$$

Thus $L_{\alpha}$ is a cuspidal representation for $\alpha=\beta(i, j), i<j$.

Corollary 3.3. We have $\operatorname{ch}_{q}^{-}\left(L_{\alpha}\right)=E_{\iota^{+}(\alpha)}^{*}$ for $\alpha \in \Phi^{+}$.

\section{Standard representations}

In this section, we use the results of the previous sections and construct all the irreducible representations of the spin quiver Hecke algebra to obtain the main result of this paper.

Recall that we have the dual canonical basis $B^{*}=\left\{b_{\mathbf{i}}^{*} \mid \mathbf{i} \in \mathcal{W}^{+}\right\}$for $\mathcal{U}_{\mathcal{A}}^{*}$. Denote the coefficient of $\mathbf{i}$ in $b_{\mathbf{i}}^{*}$ by $\kappa_{\mathbf{i}}$.

Lemma 4.1. Assume that the canonical factorization of $\mathbf{i}=\mathbf{i}_{1}^{n_{1}} \cdots \mathbf{i}_{d}^{n_{d}} \in \mathcal{W}_{\alpha}^{+}$is as in (1.25). Then $\kappa_{\mathbf{i}}=$ $\prod_{k=1}^{d} \kappa_{\mathbf{i}_{k}}^{n_{k}}\left[n_{k}\right]_{\mathbf{i}_{k}} !$

Proof. As in the proofs of [14, Proposition 39, Theorem 40] (see also [5, Theorem 7.11]), we have that $b_{\mathbf{i}}^{*}=E_{\mathbf{i}}^{*}+\sum_{\mathbf{j} \prec \mathbf{i}} \gamma_{\mathbf{i} \mathbf{j}} E_{\mathbf{j}}^{*}$. Since $\max \left(E_{\mathbf{i}}^{*}\right)=\max \left(E_{\mathbf{i}}\right)=\mathbf{i}$, it suffices to compute the coefficient of $\mathbf{i}$ in $E_{\mathbf{i}}^{*}=$ $(-1)^{\xi(\mathbf{i})} q^{s(\mathbf{i})}\left(E_{\mathbf{i}_{d}}^{*}\right)^{\diamond n_{d}} \diamond \cdots \diamond\left(E_{\mathbf{i}_{1}}^{*}\right)^{\diamond n_{1}}$. Since $\mathbf{i}_{k} \in \mathcal{L}^{+}$, we have that $E_{\mathbf{i}_{k}}^{*}=b_{\mathbf{i}_{k}}^{*}$ for all $k=1, \cdots, d$, and the coefficient of $\mathbf{i}_{k}$ in $E_{\mathbf{i}_{k}}^{*}$ is $\kappa_{\mathbf{i}_{k}}$. Now, by Corollary 1.23 , we have that the coefficient of $\mathbf{i}$ in $\mathbf{i}_{d}^{\diamond n_{d}} \diamond \cdots \diamond \mathbf{i}_{1}^{\diamond n_{1}}$ is $(-1)^{\xi(\mathbf{i})} q^{-s(\mathbf{i})} \prod_{k=1}^{d}\left[n_{k}\right]_{\mathbf{i}_{k}}$ !. Hence the coefficient of $\mathbf{i}$ in $E_{\mathbf{i}}^{*}$ is $\prod_{k=1}^{d} \kappa_{\mathbf{i}_{k}}^{n_{k}}\left[n_{k}\right]_{\mathbf{i}_{k}}$ !.

Lemma 4.2. Let $\beta \in \Phi^{+}$and $m \in \mathbb{Z}_{>0}$. Then $L_{\beta}^{\circ m}$ is irreducible with highest weight $\iota^{+}(\beta)^{m}$, and $\operatorname{ch}_{q}^{-}\left(L_{\beta}^{\circ m}\right)=$ $(-1)^{\xi\left(\iota^{+}(\beta)^{m}\right)} q^{-s\left(\iota^{+}(\beta)^{m}\right)} b_{\iota^{+}(\beta)^{m}}^{*}$.

Proof. Recall that since $\iota^{+}(\beta) \in \mathcal{L}^{+}$we have $b_{\iota^{+}(\beta)}^{*}=E_{\iota^{+}(\beta)}^{*}$. By Corollary 3.3 we have $\operatorname{ch}_{q}^{-}\left(L_{\beta}\right)=E_{\iota^{+}(\beta)}^{*}$. From Proposition 2.13, Lemma 1.28 and Corollary 1.31, it follows that

$$
\begin{aligned}
\operatorname{ch}_{q}^{-}\left(L_{\beta}^{\circ m}\right) & =\left(\operatorname{ch}_{q}^{-}\left(L_{\beta}\right)\right)^{\diamond m} \\
& =\left(E_{\iota^{+}(\beta)}^{*}\right)^{\diamond m} \\
& =(-1)^{\xi\left(\iota^{+}(\beta)^{m}\right)} q^{-s\left(\iota^{+}(\beta)^{m}\right)} E_{\iota^{+}(\beta)^{m}}^{*}=(-1)^{\xi\left(\iota^{+}(\beta)^{m}\right)} q^{-s\left(\iota^{+}(\beta)^{m}\right)} b_{\iota^{+}(\beta)^{m}}^{*} .
\end{aligned}
$$

Hence all composition factors of $L_{\beta}^{\circ m}$ have highest weight $\iota^{+}(\beta)^{m}$. Recall that the map $\operatorname{ch}_{q}^{-}$is injective by Proposition 2.14. Since $\left\{b_{\mathbf{i}}^{*}|| \mathbf{i} \mid=m \beta\right\}$ is a basis of $\left(\mathcal{U}_{\mathcal{A}}^{*}\right)_{m \beta}$ and $\boldsymbol{\iota}^{+}(\beta)^{m}$ is the smallest dominant word in $\mathcal{W}_{m \beta}^{+}$, the representation $L_{\beta}^{\circ m}$ is irreducible.

Consider $\mathbf{i} \in \mathcal{W}_{\alpha}^{+}$and write it in the form of the canonical factorization $\mathbf{i}=\mathbf{i}_{1}^{n_{1}} \cdots \mathbf{i}_{d}^{n_{d}}$. Let $\beta_{k}=\left|\mathbf{i}_{k}\right|$ for $k=1, \ldots, d$, and define the standard module $\Delta(\mathbf{i})$ of highest weight $\mathbf{i} \in \mathcal{W}_{\alpha}^{+}$over the algebra $\mathcal{H}^{-}(\alpha)$ by

$$
\Delta(\mathbf{i}):=\Pi^{\xi(\mathbf{i})}\left(L_{\beta_{1}}^{\circ n_{1}} \circ L_{\beta_{2}}^{\circ n_{2}} \circ \cdots \circ L_{\beta_{d}}^{\circ n_{d}}\right)\{s(\mathbf{i})\}
$$

where $\xi(\mathbf{i})$ and $s(\mathbf{i})$ are defined in (1.22). 
Lemma 4.3. Let $\mathbf{i} \in \mathcal{W}^{+}$. Then the highest weight of $\Delta(\mathbf{i})$ is $\mathbf{i}$, and $\operatorname{dim}_{q}^{-}(\mathbf{i} \Delta(\mathbf{i}))=\kappa_{\mathbf{i}}$.

Proof. It is easy to see that $\xi(\mathbf{i})=\sum_{k=1}^{d} \xi\left(\iota^{+}\left(\beta_{k}\right)^{n_{k}}\right)$ and $s(\mathbf{i})=\sum_{k=1}^{d} s\left(\iota^{+}\left(\beta_{k}\right)^{n_{k}}\right)$. It follows from Proposition 2.13 and Lemma 1.28 that

$$
\begin{aligned}
\operatorname{ch}_{q}^{-}(\Delta(\mathbf{i})) & =(-1)^{\xi(\mathbf{i})} q^{s(\mathbf{i})} \operatorname{ch}_{q}^{-}\left(L_{\beta_{d}}\right)^{\diamond n_{d}} \diamond \cdots \diamond \operatorname{ch}_{q}^{-}\left(L_{\beta_{1}}\right)^{\diamond n_{1}} \\
& =(-1)^{\xi(\mathbf{i})} q^{s(\mathbf{i})}\left(E_{\iota^{+}\left(\beta_{d}\right)}^{*}\right)^{\diamond n_{d}} \diamond \cdots \diamond\left(E_{\iota^{+}\left(\beta_{1}\right)}^{*}\right)^{\diamond n_{1}} \\
& =E_{\mathbf{i}}^{*}
\end{aligned}
$$

Hence the highest weight of $\Delta(\mathbf{i})$ is $\max \left(E_{\mathbf{i}}^{*}\right)=\mathbf{i}$ and $\operatorname{dim}_{q}^{-}(\mathbf{i} \Delta(\mathbf{i}))=\kappa_{\mathbf{i}}$ by Lemma 4.1 .

For $\mu, \nu \in Q^{+}$, we will write $\operatorname{Hom}_{\mu, \nu}$ for $\operatorname{Hom}_{\mathcal{H}^{-}(\mu) \otimes \mathcal{H}^{-}(\nu)}$ and recall that we write $\operatorname{Hom}_{\nu}$ for $\operatorname{Hom}_{\mathcal{H}^{-}(\nu)}$. For $M \in \operatorname{Mod}^{-}(\mu) \otimes \operatorname{Mod}^{-}(\nu)$ and $N \in \operatorname{Mod}^{-}(\mu+\nu)$, we have the Frobenius reciprocity:

$$
\operatorname{Hom}_{\mu+\nu}\left(\operatorname{Ind}_{\mu, \nu}^{\mu+\nu} M, N\right) \cong \operatorname{Hom}_{\mu, \nu}\left(M, \operatorname{Res}_{\mu, \nu}^{\mu+\nu} N\right) .
$$

Proposition 4.4. Let $\mathbf{i} \in \mathcal{W}_{\alpha}^{+}, \alpha \in Q^{+}$. Then the standard module $\Delta(\mathbf{i})$ has an irreducible head, which will be denoted by $L(\mathbf{i})$, and the highest weight of $L(\mathbf{i})$ is $\mathbf{i}$.

Proof. Let $L \in \operatorname{Rep}(\alpha)$ be irreducible. If $L$ is a component of the head of $\Delta(\mathbf{i})$, then $\operatorname{Hom}_{\alpha}(\Delta(\mathbf{i}), L)$ is nonzero and equal to

$$
\operatorname{Hom}_{n_{1} \beta_{1}, \ldots, n_{d} \beta_{d}}\left(\Pi^{\xi(\mathbf{i})}\left(L_{\beta_{1}}^{\circ n_{1}} \otimes L_{\beta_{2}}^{\circ n_{2}} \otimes \cdots \otimes L_{\beta_{d}}^{\circ n_{d}}\right)\{s(\mathbf{i})\}, \operatorname{Res}_{n_{1} \beta_{1}, \ldots, n_{d} \beta_{d}}^{\alpha} L\right)
$$

by the Frobenius reciprocity. By Lemma 4.2 , the $\mathcal{H}^{-}\left(n_{1} \beta_{1}\right) \otimes \cdots \otimes \mathcal{H}^{-}\left(n_{d} \beta_{d}\right)$-module $\Pi \xi(\mathbf{i})\left(L_{\beta_{1}}^{\circ n_{1}} \otimes L_{\beta_{2}}^{\circ n_{2}} \otimes\right.$ $\left.\cdots \otimes L_{\beta_{d}}^{\circ n_{d}}\right)\{s(\mathbf{i})\}$ is irreducible and embeds into $L$. It follows from Lemma 4.3 that the multiplicity of the weight $\mathbf{i}$ in $\Pi^{\xi(\mathbf{i})}\left(L_{\beta_{1}}^{\circ n_{1}} \otimes L_{\beta_{2}}^{\circ n_{2}} \otimes \cdots \otimes L_{\beta_{d}}^{\circ n_{d}}\right)\{s(\mathbf{i})\}$ is equal to that of the weight $\mathbf{i}$ in $\Delta(\mathbf{i})$. Thus the head of $\Delta(\mathbf{i})$ is irreducible.

Now we state and prove the main result of this paper.

Theorem 4.5. Let $\alpha \in Q^{+}$. Then the set $\left\{L(\mathbf{i}) \mid \mathbf{i} \in \mathcal{W}_{\alpha}^{+}\right\}$is a complete and irredundant set of irreducible graded $\mathcal{H}^{-}(\alpha)$-modules up to isomorphism and degree shift.

Proof. By Proposition 4.4, we have constructed an irreducible module $L(\mathbf{i})$ for each $\mathbf{i} \in \mathcal{W}_{\alpha}^{+}$. Furthermore, since the highest weights are different, we have $L(\mathbf{i}) \not L(\mathbf{j})$ for $\mathbf{i} \neq \mathbf{j}$. We have the basis $B^{*}=\left\{b_{\mathbf{i}}^{*} \mid \mathbf{i} \in \mathcal{W}^{+}\right\}$ for $\mathcal{U}_{\mathcal{A}}^{*}$, and a basis of the weight space $\left(\mathcal{U}_{\mathcal{A}}^{*}\right)_{\alpha}$ is given by $\left\{b_{\mathbf{i}}^{*} \in B^{*} \mid \mathbf{i} \in \mathcal{W}_{\alpha}^{+}\right\}$. Now the assertion of the theorem follows from Corollary 2.12.

\section{Acknowledgement}

We thank Se-jin Oh for useful comments.

\section{References}

[1] Georgia Benkart, Seok-Jin Kang, Duncan Melville, Quantized enveloping algebras for Borcherds superalgebras, Trans. Am. Math. Soc. 350 (8) (1998) 3297-3319.

[2] Georgia Benkart, Seok-Jin Kang, Se-jin Oh, Euiyong Park, Construction of irreducible representations over KhovanovLauda-Rouquier algebras of finite classical type, Int. Math. Res. Not. 2014 (5) (2014) 1312-1366. 
[3] Leonid A. Bokut, Seok-Jin Kang, Kyu-Hwan Lee, Peter Malcolmson, Gröbner-Shirshov bases for Lie superalgebras and their universal enveloping algebras, J. Algebra 217 (2) (1999) 461-495.

[4] Jonathan Brundan, Alexander Kleshchev, Peter J. McNamara, Homological properties of finite type Khovanov-LaudaRouquier algebras, Duke Math. J. 163 (7) (2014) 1353-1404.

[5] Sean Clark, David Hill, Weiqiang Wang, Quantum shuffles and quantum supergroups of basic type, preprint, arXiv:1310.7523v3, 2014.

[6] David Hill, George Melvin, Damien Mondragon, Representations of quiver Hecke algebras via Lyndon bases, J. Pure Appl. Algebra 216 (5) (2012) 1052-1079.

[7] David Hill, Weiqiang Wang, Categorification of quantum Kac-Moody superalgebras, Trans. Am. Math. Soc. 367 (2015) 1183-1216.

[8] Seok-Jin Kang, Masaki Kashiwara, Se-jin Oh, Supercategorification of quantum Kac-Moody algebras, Adv. Math. 242 (2013) 116-162.

[9] Seok-Jin Kang, Masaki Kashiwara, Se-jin Oh, Supercategorification of quantum Kac-Moody algebras II, Adv. Math. 265 (2014) 169-240.

[10] Seok-Jin Kang, Masaki Kashiwara, Shunsuke Tsuchioka, Quiver Hecke superalgebras, preprint, arXiv:1107.1039, 2011.

[11] Khovanov Mikhail, Aaron D. Lauda, A diagrammatic approach to categorification of quantum groups I, Represent. Theory 13 (09) (2009) 309-347.

[12] Alexander Kleshchev, Arun Ram, Representations of Khovanov-Lauda-Rouquier algebras and combinatorics of Lyndon words, Math. Ann. 349 (4) (2011) 943-975.

[13] Aaron D. Lauda, Monica Vazirani, Crystals from categorified quantum groups, Adv. Math. 228 (2) (2011) $803-861$.

[14] Bernard Leclerc, Dual canonical bases, quantum shuffles and q-characters, Math. Z. 246 (4) (2004) $691-732$.

[15] Guy Melançon, Combinatorics of Hall trees and Hall words, J. Comb. Theory, Ser. A 59 (2) (1992) $285-308$.

[16] Raphaël Rouquier, 2-Kac-Moody algebras, arXiv:0812.5023 [math.RT], 2008. 